\title{
Chemical Constituents and Health Benefits of Four Chinese Plum Species
}

\author{
Wei Liu, ${ }^{1}$ Guangming Nan, ${ }^{1}$ Muhammad Farrukh Nisar $\mathbb{D}^{2,3,4}$ and Chunpeng Wan ${ }^{3}{ }^{3}$ \\ ${ }^{1}$ Key Lab of Natural Product Chemistry and Application at Universities of Education Department of Xinjiang Uygur \\ Autonomous Region, Yili Normal University, Yining 835000, China \\ ${ }^{2}$ Key Laboratory of Crop Physiology, Ecology and Genetic Breeding, Ministry of Education, Jiangxi Agricultural University, \\ Nanchang 330045, Jiangxi, China \\ ${ }^{3}$ College of Agronomy, Jiangxi Agricultural University, \\ Jiangxi Key Laboratory for Post-harvest Technology and Nondestructive Testing of Fruits \& Vegetables, \\ Collaborative Innovation Center of Post-harvest Key Technology and Quality Safety of Fruits and Vegetables in Jiangxi Province, \\ Nanchang 330045, China \\ ${ }^{4}$ Department of Physiology and Biochemistry, Cholistan University of Veterinary and Animal Sciences (CUVAS), \\ Bahawalpur 63100, Pakistan
}

Correspondence should be addressed to Muhammad Farrukh Nisar; mfarrukhnisar@cuvas.edu.pk and Chunpeng Wan; chunpengwan@jxau.edu.cn

Received 23 April 2020; Revised 10 May 2020; Accepted 24 June 2020; Published 22 July 2020

Academic Editor: Quancai Sun

Copyright $\odot 2020$ Wei Liu et al. This is an open access article distributed under the Creative Commons Attribution License, which permits unrestricted use, distribution, and reproduction in any medium, provided the original work is properly cited.

\begin{abstract}
Prunus is a large genus in the Rosaceae family of flowering plants, comprising over 340 species inhabiting variable landscapes in the world. Over 500 listed phytochemicals have been isolated from this single genus so far. The present study focused four Chinese Prunus species, viz., Prunus cerasifera, Prunus domestica, Prunus salicina, and Prunus spinosa, due to their uses, demand, nutritional value, medicinal importance, and diverse biological potential. The current review article highlights the details about the active phytochemicals and various pharmacological activities already reported. Almost 212 compounds, the majority of which are flavonoids, phenolic acids, anthocyanins, and their derivatives, which have been isolated from these four Prunus species fall in different categories and are helpful to evade chronic oxidative stress-mediated diseases. A huge variation exists in the total phytochemicals composition in different Prunus species, making these species to have different biological activities in multiple disease conditions, and even the same variety growing under different edaphic conditions may have different antioxidant capacities. It is suggested to perform extensive and indepth studies to find new phytochemicals from these four Chinese Prunus species which could boost the local industry to fulfill the increasing demands.
\end{abstract}

\section{Introduction}

Prunus is a large genus (340 species) comprising variable habit of plants belonging to family Rosaceae of flowering plants. The major representatives of the genus Prunus include plums, cherries, peaches, apricots, and almonds [1]. Over 500 listed phytochemicals have been isolated from this single genus so far from this genus. In the past few decades, plums have been explained as health-promoting foods due to their anti-inflammatory actions, improving neurological disorders and strong antioxidant nature mainly because of phenolic compounds and anthocyanins [2].

Prunus species are rich in biologically active ingredients, e.g., apigenin bears strong anticancer properties as a study by Jiang and colleagues showed that it inhibits hypoxia-inducible factor-1 (HIF-1) and vascular endothelial growth factor (VEGF) expression in human ovarian cancer cells [3]. Moreover, Apigenin inhibited tumorigenesis as examined by Matrigel plug assay and chorioallantoic membrane assay (CAM assay) [3]. Similarly, another active ingredient luteolin in Prunus species, which is also reported to show 
strong anticancer activity by inhibiting the growth and invasion of prostate cancer (PC3) cells, is used to check lung metastasis in an in vivo study, and hence, it is highlighted that luteolin targets E-cadherin and may be a useful way to cure invasive prostrate cancer [4]. Chrysin shows anticancer effects by potentially inhibiting HIF-1 $\alpha$ [5], while kaempferol, quercetin, and multiforin extracted from Prunus species showed strong antioxidant potential in the DPPH radical scavenging assay [6-8]. Traditionally, plums are dried and processed for a prolonged shelf life of plums. Considering the dried fruits in terms of the phytochemical constituents, the presence of antioxidants has been reported to be the maximum compared with other dried fruits or vegetables [9].

Reactive oxygen/nitrogen species (ROS/RNS) are important physiological molecules involved, among others, in cell signaling and host defense [10]. However, an uncontrolled and excessive ROS/RNS production may overwhelm the antioxidant capacity of the cells and lead to deleterious oxidative stress, which is implicated in the pathogenesis of numerous chronic diseases [11]. Various polyphenolic compounds or plant extracts, as natural antioxidants, are regarded as a potential treatment option in prophylaxis and adjunctive therapy of CVD, and their mechanisms of action, including possible interactions with ROS/RNS, are closely looked into the restriction of onset of oxidative stress-mediated disease [12].

1.1. Aims and Objectives. As the genus Prunus is one of the largest ones, there is no authentic and detailed study describing the biological activities of its constituents in four Chinese Prunus species, viz., $P$. cerasifera, $P$. domestica, $P$. salicina, and $P$. spinosa. Hence, the current review article will highlight the details about the active phytochemicals and various pharmacological activities already reported.

\section{Chemical Constituents of Four Plum Species}

Almost 212 compounds, the majority of which were flavonoids and phenolic acids, isolated from different Prunus species have been classified under different categories and summarized in Tables 1-3.

2.1. Flavonoids. Approximately 113 flavonoids compounds (Table 1, Figure 1) including 9 anthocyanins (1-9), 33 proanthocyanins (10-42), 55 flavone and flavonols (43-92, 105-106, 110-111, 113), 13 dihydroflavonols (93-104), 3 isoflavonoids (107-109), and 1 dihydrochalcone (112) were identified from $P$. cerasifera, $P$. domestica, $P$. salicina, and $P$. spinosa.

2.2. Phenolic Acids. The analysis of phenolic acids (Table 2, Figure 2) indicated that chlorogenic acid was the predominant compound. Seven benzoic acid derivatives (114-120, 140, 141), 7 cinnamic acid derivatives $(121-127,148,149), 6$ caffeoylquinic acid derivatives (128-133), 2 feruloylquinic acid derivatives $(\mathbf{1 3 4}, \mathbf{1 3 5}), 3$ comaroylquinic acid derivatives (136-139), 2 shikimic acid $(\mathbf{1 4 2}, \mathbf{1 4 3})$, ellagic acid (145), 2 propionic acid $(146,147), 2$ abscisic acid $(152,153)$, and 3 abscisic acid derivatives (154-158) were identified from $P$. cerasifera, $P$. domestica, $P$. salicina, and $P$. spinosa.

2.3. Others. A total of 16 individual phenolic compounds have been identified from $P$. domestica. The major components identified and quantitated by HPLC-ESI-MS comprise $(163,166,167,180)$, along with a neolignane $(171)$, two guajacyl-glycerin-coniferyl aldehyde isomers $(\mathbf{1 7 2}, \mathbf{1 7 3})$ and dehydro-diconiferyl aldehyde (174). Three cyanogenic glycosids (167-169) were identified from P. spinosa. Four organic acids (175-178) and vitamin C (179) contents of three plum species ( $P$. cerasifera, $P$. domestica, and $P$. spinosa.) were determined by the HPLC method. Two carbaldehyde derivatives (180, 181), 3 steroids (185-187), 4 terpenoids (188-191, 204, 205), 8coumarin derivatives (193201), 3 cinnamic acid derivatives $(202,203,209)$, and 2 glucosyl terpenates $(\mathbf{2 0 5}, \mathbf{2 0 8})$ were identified from different Prunus species (Table 3, Figure 3).

\section{Biological Activities of Four Plum Species}

Various compounds have been isolated from different Prunus species and classified under different categories, viz., polyphenols, flavonoids, anthocyanins, alkaloids, and terpenes. The pharmacological properties of all four Chinese plum species have been discussed in the next sections.

3.1. Prunus cerasifera. Cherry plum (P. cerasifera Ehrh. and Rosaceae) or "Myrobalan plum" is a well-known medicinal plant and is a rich source of polyphenolics, anthocyanins, carotenoids, flavonoids, various organic acids, aromatic compounds, tannins, minerals, vitamins, and antioxidant compounds $[13,71]$. The Myrobalan plum fruits are rich in health-promoting phytochemicals that help prevent the onset of different diseases. The $P$. cerasifera fruit has strong antibacterial and antifungal potential of pathogenic class for medical sciences and agricultural product-related pathogens [72]. The purple Myrobalan plum fruit peels have high amounts of anthocyanins and phenolic compounds due to which it showed high antioxidant activity [24]. Considerable differences have also been reported for $P$. cerasifera accessions with regard to total phenolics and antioxidant activity [73]. Another report highlighted that tannins found in $P$. cerasifera have high antioxidant properties and showed potential inhibition of tyrosinase activity and, thus, could be used as a strong inhibitor for the onset of melanogenesis [29]. Gunduza and Saracoglu studied various fruit characteristics (total phenolics and antioxidant activity) in different accessions of $P$. cerasifera from Turkey and reported considerable variation for these characters among the accessions, but the phenolic contents are comparable to many other plum species [73]. The antioxidant capability of plums mainly depends on the level of ripening, and this trend is entirely opposite to that of the phenolic contents [74]. The leaves of $P$. cerasifera are enriched with biologically active ingredients, notably tannins, flavonoids, and phenolic acids, 
TABle 1: Flavonoids compounds isolated and identified from different Prunus.

\begin{tabular}{|c|c|c|c|}
\hline No. & Compounds & Sources & Ref. \\
\hline 1 & Cyanidin-3-galactoside & $\begin{array}{l}\text { P. cerasifera Ehrh., P. salicina } \\
\text { Lindl. }\end{array}$ & [13-17] \\
\hline 2 & Cyanidin-3-glucoside & $\begin{array}{c}\text { P. cerasifera Ehrh., P. salicina } \\
\text { Lindl., P. domestica L., P. spinosa } \\
\text { L. }\end{array}$ & {$[13-15,18-27]$} \\
\hline 3 & Cyanidin-3-rutinoside & $\begin{array}{c}\text { P. cerasifera Ehrh., P. salicina } \\
\text { Lindl., P. domestica L., P. spinosa } \\
\text { L. }\end{array}$ & [13-27] \\
\hline 4 & Cyanidin-3-acetylglucoside & P. cerasifera Ehrh. & [13] \\
\hline 5 & Cyanidin-3-xyloside & $\begin{array}{c}\text { P. cerasifera Ehrh., P. domestica } \\
\text { L. }\end{array}$ & {$[13,23,27]$} \\
\hline 6 & Cyanidin-3-pentoside & P. spinosa $L$. & {$[21,28]$} \\
\hline 7 & Peonidin-3-rutinoside & P. spinosa L., P. domestica $L$. & {$[18,21-25,27]$} \\
\hline 8 & Peonidin-3-glucoside & P. spinosa L., P. domestica $L$. & {$[19-25,27]$} \\
\hline 9 & Peonidin-3-acetylglucoside & $\quad$ P. spinosa L. & [21] \\
\hline 10 & Afzelechin & P. cerasifera Ehrh., P. spinosa $L$. & {$[29,30]$} \\
\hline 11 & Epiafzelechin & P. cerasifera Ehrh., P. spinosa L. & {$[29-31]$} \\
\hline 12 & $(+)$-Catechin & $\begin{array}{l}\text { P. salicina Lindl., P. domestica L., } \\
P . \text { cerasifera Ehrh., P. spinosa } L .\end{array}$ & {$[8,15,16,24,29-39]$} \\
\hline 13 & (-)-Epicatechin & $\begin{array}{l}\text { P. salicina Lindl., P. domestica L., } \\
\text { P. spinosa L. }\end{array}$ & {$[15,16,24,29-32,35,36,38-42]$} \\
\hline 14 & Epigallocatechin & P. spinosa $L$ & {$[30]$} \\
\hline 15 & Gallocatechin & P. spinosa $L$. & {$[30]$} \\
\hline 16 & Procyanidin $\mathrm{B} 1$ & P. salicina Lindl., P. domestica L. & {$[15,16,35,36,39]$} \\
\hline 17 & Procyanidin B2 & P. salicina Lindl., P. domestica L. & {$[15,16,28,35,36,39]$} \\
\hline 18 & Epicatechin 3-O-gallate & P. domestica $L$. & [39] \\
\hline 19 & Ent-epicatechin- $(2 \alpha \longrightarrow O \longrightarrow 7 ; 4 \alpha \longrightarrow 8)$-catechin & P. spinosa $L$. & {$[30,31,43]$} \\
\hline 20 & $\begin{array}{l}\text { Ent-epicatechin- }(2 \alpha \longrightarrow O \longrightarrow 7 ; 4 \alpha \longrightarrow 8) \text {-catechin } \\
5,7,11,12,5^{\prime} 11^{\prime}, 12^{\prime} \text {-heptamethyl ether-3,3, } 3^{\prime} \text {-diacetate }\end{array}$ & P. spinosa $L$. & {$[31,43]$} \\
\hline 21 & Ent-epicatechin- $(2 \alpha \longrightarrow O \underset{\text { acetate }}{\longrightarrow} 7 ; 4 \alpha \longrightarrow 8)$-catechin-nona- & P. spinosa $L$. & [43] \\
\hline 22 & Ent-epigallocatechin-( $2 \alpha \longrightarrow O \longrightarrow 7 ; 4 \alpha \longrightarrow 8)$-epicatechin & P. spinosa $L$. & {$[30]$} \\
\hline 23 & Ent-epicatechin-( $2 \alpha \longrightarrow O \longrightarrow 7 ; 4 \alpha \longrightarrow 8)$-epiafzelechin & P. spinosa $L$. & {$[30,43,44]$} \\
\hline 24 & $\begin{array}{c}\text { Ent-epicatechin- }(2 \alpha \longrightarrow O \longrightarrow 7 ; 4 \alpha \longrightarrow 8) \text {-epiafzelechin } \\
7,12,5^{\prime}, 12^{\prime} \text {-tetramethyl ether }\end{array}$ & P. spinosa $L$. & {$[44]$} \\
\hline 25 & 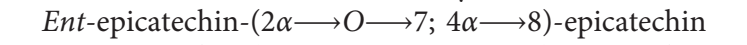 & P. spinosa L., P. spinosa $L$. & {$[32,36,43]$} \\
\hline 26 & $\begin{array}{l}\text { Ent-epicatechin- }(2 \alpha \longrightarrow O \longrightarrow 7 ; 4 \alpha \longrightarrow 8) \text {-epicatechin } \\
5,7,11,12,5^{\prime} 11^{\prime}, 12^{\prime} \text {-heptamethyl ether-3,3'-diacetate }\end{array}$ & $P$. spinosa $L$. & {$[43]$} \\
\hline 27 & $\begin{array}{l}\text { Ent-epicatechin- }(2 \alpha \longrightarrow O \longrightarrow 7 ; 4 \alpha \longrightarrow 8) \text {-epicatechin } \\
7,11,12,5^{\prime} 11^{\prime}, 12^{\prime} \text {-hexamethyl ether-3,5,3'-triacetate }\end{array}$ & P. spinosa $L$. & {$[43]$} \\
\hline 28 & 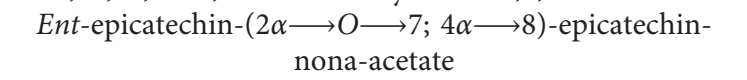 & P. spinosa $L$. & {$[43]$} \\
\hline 29 & Ent-epiafzelechin-( $2 \alpha \longrightarrow O \longrightarrow 7 ; 4 \alpha \longrightarrow 8)$-epicatechin & P. spinosa $L$. & {$[30,32,43-45]$} \\
\hline 30 & $\begin{array}{c}\text { Ent-epiafzelechin- }(2 \alpha \longrightarrow O \longrightarrow 7 ; 4 \alpha \longrightarrow 8) \text {-epiafzelechin } \\
\quad 7,12,5^{\prime}, 12^{\prime} \text {-tetramethyl ether-3,5,3'-triacetate }\end{array}$ & P. spinosa $L$. & {$[44]$} \\
\hline 31 & $\begin{array}{c}\text { Ent-epiafzelechin- }(2 \alpha \longrightarrow O \longrightarrow 7 ; 4 \alpha \longrightarrow 8) \text {-epiafzelechin- } \\
\text { hepta-acetate }\end{array}$ & P. spinosa $L$. & {$[43,44]$} \\
\hline 32 & $\begin{array}{c}\text { Ent-epiafzelechin- }(2 \alpha \longrightarrow O \longrightarrow 7 ; 4 \alpha \longrightarrow 8) \text {-epicatechin } \\
\qquad 7,12,5^{\prime}, 11^{\prime}, 12^{\prime} \text {-pentamethyl ether }\end{array}$ & P. spinosa $L$. & {$[44]$} \\
\hline 33 & $\begin{array}{c}\text { Ent-epiafzelechin- }(2 \alpha \longrightarrow O \longrightarrow 7 ; 4 \alpha \longrightarrow 8) \text {-epicatechin- } \\
\text { octa-acetate }\end{array}$ & P. spinosa $L$. & {$[44]$} \\
\hline 34 & $\begin{array}{l}\text { Ent-epiafzelechin }-(2 \alpha \longrightarrow O \longrightarrow 7 ; 4 \alpha \longrightarrow 8) \text {-epicatechin } \\
\quad 5,7,12,5^{\prime} 11^{\prime}, 12^{\prime} \text {-hexamethyl ether-3,3 } 3^{\prime} \text {-diacetate }\end{array}$ & $P$. spinosa $L$. & {$[43]$} \\
\hline 35 & $\begin{array}{l}\text { Ent-epiafzelechin- }(2 \alpha \longrightarrow O \longrightarrow 7 ; 4 \alpha \longrightarrow 8) \text {-epicatechin } \\
\quad 7,12,5^{\prime} 11^{\prime}, 12^{\prime} \text {-pentamethyl ether-3,5,3'-triacetate }\end{array}$ & P. spinosa $L$. & {$[43]$} \\
\hline 36 & $\begin{array}{c}\text { Ent-epiafzelechin- }(2 \alpha \longrightarrow O \longrightarrow \\
O \text {-gallate }\end{array}$ & $P$. spinosa $L$. & {$[30]$} \\
\hline 37 & Ent-epicatechin- $(2 \alpha \longrightarrow O \underset{O \text {-gallate }}{\longrightarrow} 7 ; 4 \alpha \longrightarrow 8)$-epicatechin- $3^{\prime}$ - & P. spinosa $L$. & {$[30]$} \\
\hline 38 & Ent-epiafzelechin- $(2 \alpha \longrightarrow O \longrightarrow 7 ; 4 \alpha \longrightarrow 8)$-catechin & P. spinosa $L$. & {$[30,45]$} \\
\hline
\end{tabular}


TABle 1: Continued.

\begin{tabular}{|c|c|c|c|}
\hline No. & Compounds & Sources & Ref. \\
\hline 39 & $\begin{array}{c}\text { Ent-epiafzelechin- }(2 \alpha \longrightarrow O \longrightarrow \\
\text { acetate }\end{array}$ & P. spinosa $L$. & {$[43]$} \\
\hline 40 & Proanthocyanidin oligomer & P. domestica $L$. & {$[46]$} \\
\hline 41 & Epicatechin- $4,8^{\prime}$-epicatechin $-4^{\prime}, 8^{\prime \prime}$-epicatechin & P. domestica $L$. & {$[36]$} \\
\hline 42 & $\begin{array}{c}\text { Epicatechin- } 4,8^{\prime} \text {-epicatechin- }\left(2^{\prime} \alpha \longrightarrow O \longrightarrow 7^{\prime \prime}\right. \\
\left.\qquad 4^{\prime} \alpha \longrightarrow 8^{\prime \prime}\right) \text {-epicatechin }\end{array}$ & P. domestica $L$. & {$[36]$} \\
\hline 43 & Quercetin & $\begin{array}{l}\text { P. spinosa } L ., P \text {. domestica } L ., P \text {. } \\
\text { cerasifera Ehrh. }\end{array}$ & {$[28,30-32,36,38,47-50]$} \\
\hline 44 & Quercetin-3-O- $\alpha$-L-arabinofuranoside & P. spinosa $L ., P$. spinosa $L$. & {$[32,33,47]$} \\
\hline 45 & Quercetin-3-rutinoside & $\begin{array}{l}\text { P. salicina Lindl., P. domestica L., } \\
P . \text { cerasifera Ehrh., P. spinosa } L .\end{array}$ & $\begin{array}{l}{[8,15,16,21,22,24,26,28,30} \\
35-39,42,49-55]\end{array}$ \\
\hline 46 & Quercetin-3-O-glucoside & $\begin{array}{l}\text { P. salicina Lindl., P. domestica L., } \\
\text { P. spinosa L. }\end{array}$ & {$[15-17,19,21,22,24,26,28,35,36]$} \\
\hline 47 & Quercetin-3-O-xyloside & $\begin{array}{c}\text { P. salicina Lindl., P. cerasifera } \\
\text { Ehrh. }\end{array}$ & {$[15-17,56]$} \\
\hline 48 & Quercetin-3-O-arabinoside & $\begin{array}{l}\text { P. salicina Lindl., P. domestica L., } \\
\text { P. cerasifera Ehrh. }\end{array}$ & {$[15,16,24,56]$} \\
\hline 49 & Quercetin-3-O-rhamnoside & $\begin{array}{l}\text { P. salicina Lindl., P. domestica L., } \\
\text { P. cerasifera Ehrh. }\end{array}$ & {$[15,16,28,32,35,36,56]$} \\
\hline 50 & Quercetinl-7-O- $\alpha$-L-rhamnopyransoide & P. domestica $L$ & {$[32]$} \\
\hline 51 & Quercetin-3-O- $\beta$-D-galactoside & $\begin{array}{c}\text { P. salicina Lindl., P. domestica L., } \\
\text { P. domestica L., P. cerasifera } \\
\text { Ehrh. }\end{array}$ & {$[17,19,24,26,28,32,35,36,56]$} \\
\hline 52 & Quercetin-3-O- $\alpha$-D-xylopyranoside & P. domestica $L$. & {$[32]$} \\
\hline 53 & Quercetin-3-O- $\alpha$-D-glucopyranoside & P. domestica $L$. & {$[32]$} \\
\hline 54 & Quercetin-pentoside & P. domestica $L$. & {$[28,36,39]$} \\
\hline 55 & Quercetin-pentoside-rhamnoside & P. domestica $L$. & [36] \\
\hline 56 & Quercetin pentosyl-hexoside & P. salicina Lindl., P. domestica $L$. & {$[15,16,39]$} \\
\hline 57 & Quercetin pentosyl-pentoside & P. salicina Lindl. & {$[15,16,39]$} \\
\hline 58 & Quercetin-acetylhexoside & P. salicina Lindl., P. domestica L. & {$[15,16,39]$} \\
\hline 59 & Quercetin-deoxyhexose & P. domestica $L$. & [39] \\
\hline 60 & $\begin{array}{c}\text { Quercetin-3-O- }\left(4^{\prime \prime}-O-\beta \text {-D-glucopyranosyl }\right)-\alpha \text {-L- } \\
\text { rhamnopyransoide }\end{array}$ & P. domestica $L$. & {$[32]$} \\
\hline 61 & $\begin{array}{l}\text { Quercetin-3-O-(6"-O- } \alpha \text {-L-rhamnopyranosyl)- } \beta \text {-D- } \\
\text { glucopyranoside(rutin) }\end{array}$ & P. spinosa $L$. & {$[32]$} \\
\hline 62 & $\begin{array}{c}\text { Quercetin-3-O- }\left(2^{\prime \prime}-O-\beta \text {-D-glucopyranosyl }\right)-\alpha-\mathrm{L}- \\
\text { arabinofuranoside }\end{array}$ & P. spinosa $L$. & {$[32,33,47]$} \\
\hline 63 & Hyperin & P. cerasifera Ehrh. & {$[50]$} \\
\hline 64 & Kaempferol & $\begin{array}{l}\text { P. spinosa } L ., P . \text { domestica } L ., P . \\
\text { spinosa } L ., P . \text { cerasifera Ehrh. }\end{array}$ & {$[30-33,47-50,57,58]$} \\
\hline 65 & Apigenin & P. cerasifera Ehrh. & {$[50]$} \\
\hline 66 & Kaempferol-3-O- $\alpha$-D-glucoside & P. cerasifera Ehrh. & {$[50,56]$} \\
\hline 67 & Kaempferol-3,7-di-O- $\alpha$-L-rhamnopyranoside & P. spinosa $L$. & {$[30-32,47]$} \\
\hline 68 & Kaempferol-7-O- $\alpha$-L-rhamnopyransoide & P. spinosa $L$. & {$[32,33,47]$} \\
\hline 69 & $\begin{array}{l}\text { Kaempferol-3-O- } \alpha \text {-L-arabinofuranoside-7-O- } \alpha \text {-L- } \\
\text { rhamnopyransoide }\end{array}$ & P. spinosa $L$. & {$[32,33,47]$} \\
\hline 70 & Kaempferol-3-O- $\alpha$-L-arabinofuranoside & P. spinosa L., P. cerasifera Ehrh. & {$[30,32,33,47,56]$} \\
\hline 71 & Kaempferol-3-O-arabinoside-7-O-rhamnoside & P. spinosa $L$. & {$[30,32]$} \\
\hline 72 & $\begin{array}{l}\text { Kaempferol-3-O- } \alpha \text {-D-xylopyranoside-7-O- } \alpha \text {-L- } \\
\text { rhamnopyransoide(lepidoside) }\end{array}$ & P. spinosa $L$. & {$[32,33]$} \\
\hline 73 & Kaempferol-3-O- $\alpha$-D-xylopyranoside & P. spinosa L., P. cerasifera Ehrh. & {$[32,56]$} \\
\hline 74 & Kaempferol-3-O- $\alpha$-L-rhamnopyransoide & P. spinosa L., P. cerasifera Ehrh. & {$[32,33,56]$} \\
\hline 75 & $\begin{array}{l}\text { Kaempferol-3-O- }\left(2^{\prime \prime}-\mathrm{E}-\mathrm{p} \text {-conmaroyl }\right)-\alpha-\mathrm{L}- \\
\text { arabinofuranoside }\end{array}$ & P. spinosa $L$. & {$[32]$} \\
\hline 76 & Kaempferol-3-O-rutinoside & P. spinosa $L$. & {$[21,28]$} \\
\hline 77 & $\begin{array}{c}\text { Kaempferol-3-O- } \alpha \text {-L-arabinopyranoside-7-O- } \alpha \text { - } \mathrm{L}- \\
\text { rhamnopyransoide }\end{array}$ & P. spinosa $L$. & {$[33]$} \\
\hline 78 & $5,4^{\prime}$-Dihydroxyflavone-7-O- $\alpha$-D-glucoside & P. domestica $L$. & [59] \\
\hline 79 & Kaempferol-hexoside & P. domestica $L$. & {$[28,39]$} \\
\hline 80 & Kaempferol-pentoside-rhamnoside & P. domestica $L$. & {$[28]$} \\
\hline
\end{tabular}


TABle 1: Continued.

\begin{tabular}{|c|c|c|c|}
\hline No. & Compounds & Sources & Ref. \\
\hline 81 & Kaempferol-pentoside & P. domestica $L$. & {$[28]$} \\
\hline 82 & $\begin{array}{c}\text { Kaempferol-3-O- }\left(4^{\prime \prime}-O-\beta \text {-D-glucopyranosyl }\right)-\alpha-\mathrm{L} \text { - } \\
\text { rhamnopyransoide }\end{array}$ & $P$. spinosa $L$. & {$[32]$} \\
\hline 83 & $\begin{array}{l}\text { Kaempferol-3-O-(2"-E-p-conmaroyl)- } \alpha \text {-L- } \\
\text { arabinofuranoside-7-O- } \alpha \text {-L-rhamnopyransoide }\end{array}$ & P. spinosa $L$. & {$[32,33,47]$} \\
\hline 84 & Myricetin & P. salicina Lindl., P. domestica $L$. & {$[20,35,40,48,49]$} \\
\hline 85 & Luteolin & P. spinosa L., P. cerasifera Ehrh. & {$[41,50]$} \\
\hline 86 & Luteolin- $4^{\prime}-O-\alpha$-D-glucoside & P. cerasifera Ehrh. & {$[50]$} \\
\hline 87 & Prudomestin & P. domestica $L$. & {$[60]$} \\
\hline 88 & 3,5,7-Trihydroxy-4'-methoxyflavanone & P. domestica $L$. & {$[57]$} \\
\hline 89 & Isorhamnetin-3-O-rutinoside & P. domestica $L$. & {$[24,28,35]$} \\
\hline 90 & Isorhamnetin-3-O-glucoside & P. domestica $L$. & {$[24,26]$} \\
\hline 91 & Isorhamnetin-3-O-galactoside & P. domestica $L$. & {$[26]$} \\
\hline 92 & Quercetin- $4^{\prime}-O-\alpha$-D-glucoside & P. cerasifera Ehrh. & {$[50]$} \\
\hline 93 & Hesperetin & P. salicina Lindl. & {$[40]$} \\
\hline 94 & Isosakuranetin & P. domestica L. & {$[60]$} \\
\hline 95 & Dihydrokaempferide & P. domestica $L$. & {$[60]$} \\
\hline 96 & Naringenin & P. domestica $L$. & {$[60]$} \\
\hline 97 & 3,5,7-Trihydroxy- $8,4^{\prime}$-dimethoxyflavanone & P. domestica $L$. & {$[58,60]$} \\
\hline 98 & 3,5,7-Trihydroxy-6,4'-dimethoxyflavanone & P. domestica $L$. & {$[59,60]$} \\
\hline 99 & 5,7,4'-Trihydroxy-3-methoxyflavanone. & P. domestica $L$. & {$[60]$} \\
\hline 100 & $7,4^{\prime}$-Dimethylaromadendrin & P. domestica $L$. & {$[57]$} \\
\hline 101 & 5,7-Dihydroxy-4'-methoxy-dihydroflavonol & P. domestica $L$. & [57] \\
\hline 102 & 5,7-Dihydroxy- $8,4^{\prime}$-dimeth-oxyllavonol & P. domestica $L$. & {$[57]$} \\
\hline 103 & Dihydrokaempferol-3-O- $\alpha$-L-rhamnoside & P. cerasifera Ehrh. & {$[56]$} \\
\hline 104 & Dihydrokaempferol-3-O- $\alpha$-D-glucoside & P. cerasifera Ehrh. & {$[56]$} \\
\hline 105 & Prudomestiside A & P. domestica $L$. & [59] \\
\hline 106 & Prudomestiside B & P. domestica $L$. & [59] \\
\hline 107 & Purunuside A & P. domestica $L$. & {$[61]$} \\
\hline 108 & Purunuside B & P. domestica $L$. & {$[61]$} \\
\hline 109 & Purunuside C & P. domestica $L$. & {$[61]$} \\
\hline 110 & Prunusins A & P. domestica $L$. & {$[58]$} \\
\hline 111 & Prunusins B & P. domestica $L$. & {$[58]$} \\
\hline 112 & Phloridzin & $\begin{array}{c}\text { P. spinosa L., P. domestica L., P. } \\
\text { cerasifera Ehrh. }\end{array}$ & {$[37]$} \\
\hline 113 & $5,2^{\prime}$-Dihydroxy-7, $5^{\prime}$-dimethoxyflavanone & P. domestica $L$. & [59] \\
\hline
\end{tabular}

Table 2: Phenolic acids compounds isolated and identified from different Prunus species.

\begin{tabular}{|c|c|c|c|}
\hline No. & Compounds & Sources & Ref. \\
\hline 114 & Vanillic acid & $\begin{array}{l}\text { P. spinosa } L ., P . \\
\text { domestica } L ., P . \\
\text { cerasifera Ehrh. } \\
P . \text { spinosa } L ., P .\end{array}$ & {$[37,41,49,52,53,62,63]$} \\
\hline 115 & Protocatechuic acid & $\begin{array}{c}\text { domestica L., P. spinosa } \\
\text { L. }\end{array}$ & {$[6,30,39,41,42,48,52-54,62]$} \\
\hline 116 & p-Hydroxybenzoic acid & $\begin{array}{l}\text { P. spinosa } L ., P . \\
\text { domestica } L .\end{array}$ & {$[41,49,53]$} \\
\hline 117 & Vanillic acid- $\beta$-glucoside & P. domestica $L$. & {$[6,36]$} \\
\hline 118 & Vanillic acid- $\alpha$-D-glucopyranoside & $\begin{array}{l}\text { P. domestica } L \text {. } \\
\text { P. spinosa } L ., P \text {. }\end{array}$ & {$[52]$} \\
\hline 119 & Gallic acid & $\begin{array}{l}\text { domestica L., P. } \\
\text { cerasifera Ehrh., P. } \\
\text { spinosa L. }\end{array}$ & {$[19,30,37,49,50,53,54,62]$} \\
\hline 120 & Syringic acid & $\begin{array}{l}P . \text { spinosa } L ., P . \\
\text { domestica } L ., P . \\
\text { cerasifera Ehrh. }\end{array}$ & {$[34,37,38,41,49,53]$} \\
\hline 121 & Caffeic acid & $\begin{array}{c}P . \text { spinosa } L ., P . \\
\text { domestica } L ., P . \\
\text { cerasifera Ehrh., } P . \\
\text { spinosa } L .\end{array}$ & {$[6,8,19,20,30,32,34,37,42,48,49,51,52,54]$} \\
\hline 122 & Caffeic acid methyl ester & P. domestica $L$. & {$[52,63]$} \\
\hline
\end{tabular}


TABle 2: Continued.

\begin{tabular}{|c|c|c|c|}
\hline No. & Compounds & Sources & Ref. \\
\hline 123 & Ferulic acid & $\begin{array}{l}\text { P. spinosa } L ., P \text {. } \\
\text { domestica } L ., P \text {. } \\
\text { cerasifera Ehrh. } \\
\text { P. spinosa } L ., P \text {. }\end{array}$ & {$[37,48,49,62]$} \\
\hline 124 & $p$-Coumaric acid & $\begin{array}{l}\text { domestica L., } P . \\
\text { cerasifera Ehrh. }\end{array}$ & {$[6,8,24,32,34,37,39,41,42,49,51,52]$} \\
\hline 125 & Cinnamic acid & P. domestica $L$. & [49] \\
\hline 126 & Ferulic acid- $\beta$-D-glucopyranoside & P. domestica $L$. & {$[52,63]$} \\
\hline 127 & Coniferine & P. domestica $L$. & {$[52,63]$} \\
\hline 128 & 3-O-Caffeoylquinic acid (neochlorogenic acid) & $\begin{array}{c}\text { P. salicina Lindl., } P . \\
\text { domestica L., P. spinosa } \\
\text { L. }\end{array}$ & {$[8,15-17,21,22,24-26,28,32,33,35,36,42,48,51,53,54]$} \\
\hline 129 & 4-O-Caffeoylquinic acid & $\begin{array}{l}\text { P. spinosa } L ., P . \\
\text { domestica } L .\end{array}$ & {$[21,22,24,28,32,33,35,36,42,51-53]$} \\
\hline 130 & 5-O-Caffeoylquinic acid & $\begin{array}{l}\text { P. salicina Lindl., } P \text {. } \\
\text { domestica L., P. } \\
\text { cerasifera Ehrh., P. } \\
\quad \text { spinosa } L .\end{array}$ & {$[15,16,22,25,26,28,32,34-38,42,48,49,51-54,62,64]$} \\
\hline 131 & 3-O-Caffeoylquinic acid methyl ester & P. domestica $L$. & {$[36,52,63]$} \\
\hline 132 & 4-O-Caffeoylquinic acid methyl ester & P. domestica $L$. & {$[36,52,63]$} \\
\hline 133 & 5-O-Caffeoylquinic acid methyl ester & P. domestica $L$. & {$[36]$} \\
\hline 134 & 3-O-Feruloylquinic acid & $\begin{array}{l}\text { P. spinosa } L ., P \text {. } \\
\text { domestica } L .\end{array}$ & {$[21,32,33,35,36]$} \\
\hline 135 & 4-O-Feruloylquinic acid & P. spinosa $L$. & {$[32]$} \\
\hline 136 & 3-O-Coumaroylquinic acid & $\begin{array}{l}\text { P. spinosa } L ., P \text {. } \\
\text { domestica } L .\end{array}$ & {$[8,15,16,21,25,28,32,33,36,53]$} \\
\hline 137 & 4-O-Comaroylquinic acid & P. spinosa $L$. & {$[32]$} \\
\hline 138 & 5-O-Comaroylquinic acid & P. spinosa $L$. . & {$[32]$} \\
\hline 139 & 3-Coumaroylquinic acid methyl ester & P. domestica $L$. & [36] \\
\hline 140 & Salicylic acid & P. domestica $L$. & [49] \\
\hline 141 & 2,3-Dimethylbenzoic acid & P. domestica $L$. & [38] \\
\hline 142 & Shikimic acid & P. domestica $L$. & [39] \\
\hline 143 & 3-Caffeoylshikimic acid & $P$. domestica $L$. & {$[35,36]$} \\
\hline 144 & 2-(3', $4^{\prime}$-Dihydroxyphenyl)acetic acid & P. spinosa $L$. & [65] \\
\hline 145 & Ellagic acid & P. domestica $L$. & [49] \\
\hline 146 & 3-(4'-Hydroxyphenyl)propionic acid & $P$. spinosa $L$. & [65] \\
\hline 147 & 3-(3', $4^{\prime}$-Dihydroxyphenyl)propionic acid & P. spinosa $L$. & {$[65]$} \\
\hline 148 & Caffeoyl hexoside & P. domestica $L$. & [39] \\
\hline 149 & p-Coumaroyl-hexoside & P. domestica $L$. & [39] \\
\hline 150 & 3,4-Dihydroxybenzoyl-glucoses & P. domestica $L$. & [36] \\
\hline 151 & Rosmarinic acid & P. domestica $L$. & [49] \\
\hline 152 & Abscisic acid & P. domestica $L$. & {$[52,66]$} \\
\hline 153 & $\beta$-D-glusosylabscisate & P. domestica $L$. & {$[52,66]$} \\
\hline 154 & $\begin{array}{c}\text { Rel-5-(1R,5S-dimethyl-3R,4R,8S-trihydroxy-7- } \\
\text { oxabicyclo }(3,2,1) \text {-oct-8-yl)-3-methyl-2Z,4E- } \\
\text { pentadienoic acid }\end{array}$ & P. domestica $L$. & {$[52,66]$} \\
\hline 155 & $\begin{array}{c}\text { Rel-5-(1R,5S-dimethyl-3R,4R,8S-trihydroxy-7- } \\
\text { oxa-6-oxobicyclo }(3,2,1) \text { oct-8-yl)-3-methyl- } \\
\text { 2Z,4E-pentadienoic acid }\end{array}$ & P. domestica $L$. & {$[52,66]$} \\
\hline 156 & $\begin{array}{l}\text { Rel-5-(3S,8S-dihydroxy- } 1 R, 5 S \text {-dimethyl-7-oxa- } \\
\text { 6-oxobicyclo }(3,2,1) \text { oct-8-yl)-3-methyl-2Z,4E- } \\
\text { pentadienoic acid }\end{array}$ & P. domestica $L$. & {$[52,66]$} \\
\hline 157 & $\begin{array}{l}\text { Rel-5-(3S,8S-dihydroxy-1R,5S-dimethyl-7-oxa- } \\
\text { 6-oxobicyclo s oct-8-yl)-3-methyl-2Z,4E- } \\
\text { pentadienoic acid-3'-O- } \alpha \text {-D-glucopyranoside }\end{array}$ & P. domestica $L$. & {$[52,66]$} \\
\hline 158 & $(6 S, 9 R)$-roseoside & P. domestica $L$. & {$[52,66]$} \\
\hline
\end{tabular}


TABle 3: Others compounds isolated and identified from different Prunus.

\begin{tabular}{|c|c|c|c|}
\hline No. & Compounds & Sources & Ref. \\
\hline 159 & p-Hydroxybenzaldehyde & P. domestica $L$. & {$[53]$} \\
\hline 160 & Benzoic acid & P. spinosa $L ., P$. domestica $L$. & {$[36,38,49,53,62]$} \\
\hline 161 & Syringaldehyde & P. domestica $L$. & {$[53]$} \\
\hline 162 & Hydroxy-4-methoxybenzaldehyde & P. domestica $L$. & {$[38]$} \\
\hline 163 & Vanillin & P. spinosa $L ., P$. domestica $L$. & {$[49,53,62]$} \\
\hline 164 & Protocatechuic aldehyde & P. spinosa $L$. & {$[62]$} \\
\hline 165 & Coniferyl aldehyde & P. domestica $L$. & [53] \\
\hline 166 & Dimethoxycinnamaldehyde & P. domestica $L$. & {$[53]$} \\
\hline 167 & Amygdalin & $\begin{array}{l}\text { P. cerasifera Ehrh. P. domestica } \\
\text { L. }\end{array}$ & {$[53,67]$} \\
\hline 168 & Prunasin & P. cerasifera Ehrh. & [67] \\
\hline 169 & Sambunigrin & P. cerasifera Ehrh. & [67] \\
\hline 170 & Coniferyl aldehyde & P. domestica $L$. & {$[52]$} \\
\hline 171 & Pinoresinol- $O$ - $\alpha$-D-glucopyranoside & P. domestica $L$. & {$[52]$} \\
\hline 172 & Guajacyl-glycerin-coniferyl aldehyde-1 & P. domestica $L$. & [53] \\
\hline 173 & Guajacyl-glycerin-coniferyl aldehyde-2 & P. domestica $L$. & [53] \\
\hline 174 & Dehydro-diconiferyl aldehyde & P. domestica $L$. & [53] \\
\hline 175 & Fumaric acid & $\begin{array}{l}\text { P. spinosa L., P. domestica L., P. } \\
\text { cerasifera Ehrh. }\end{array}$ & [37] \\
\hline 176 & Malic acid & $\begin{array}{l}\text { P. spinosa L., P. domestica L., } P \text {. } \\
\text { cerasifera Ehrh. }\end{array}$ & [37] \\
\hline 177 & Succinic acid & $\begin{array}{l}\text { P. spinosa L., P. domestica L., P. } \\
\text { cerasifera Ehrh. }\end{array}$ & [37] \\
\hline 178 & Citric acid & $\begin{array}{l}\text { P. spinosa L., P. domestica L., } P \text {. } \\
\text { cerasifera Ehrh. }\end{array}$ & [37] \\
\hline 179 & $\begin{array}{ll}\text { Vitamin C } \\
\end{array}$ & $\begin{array}{c}\text { P. spinosa L., P. domestica L., } P . \\
\text { cerasifera Ehrh. }\end{array}$ & {$[37]$} \\
\hline 180 & $\begin{array}{c}\text { 2-(5-Hydroxymethyl-2 } 2^{\prime}, 5^{\prime} \text {-dioxo- } 2^{\prime}, 3^{\prime}, 4^{\prime}, 5^{\prime} \text {-tetrahydro-1 }{ }^{\prime} \mathrm{H}-1,3^{\prime} \text {-bipyrrole)- } \\
\text { carbaldehyde }\end{array}$ & P. domestica $L$. & {$[52]$} \\
\hline 181 & Hydroxymethylfurfural & P. domestica $L$. & [52] \\
\hline 182 & Benzyl- $\beta$-primeveroside & P. domestica $L$. & {$[52,63]$} \\
\hline 183 & Vanillin diglucoside & P. domestica $L$. & {$[53]$} \\
\hline 184 & 4-Amino-4-carboxychroman-2-one & P. domestica $L$. & {$[6]$} \\
\hline 185 & $\beta$-Sitosterol & $\begin{array}{l}\text { P. domestica L., P. cerasifera } \\
\text { Ehrh. }\end{array}$ & {$[50,61]$} \\
\hline 186 & Daucosterol & P. cerasifera Ehrh. & [50] \\
\hline 187 & Stigmasterol & P. cerasifera Ehrh. & {$[50]$} \\
\hline 188 & Ursolic acid & P. cerasifera Ehrh. & [50] \\
\hline 189 & Arjunolic acid & P. cerasifera Ehrh. & {$[50]$} \\
\hline 190 & Niga-ichigoside F1 & P. cerasifera Ehrh. & [50] \\
\hline 191 & Lupeol & P. cerasifera Ehrh. & [50] \\
\hline 192 & $\begin{array}{l}\text { 3-( } \alpha \text {-D-glucopyranosyloxymethyl)-2-(4-hydroxy-3-methoxyphenyl)-5-(3- } \\
\text { hydroxypropyl)-7-methoxy-(2R,3S)-dihydrobenzofuran }\end{array}$ & P. domestica $L$. & {$[52,66]$} \\
\hline 193 & 5-Hydroxy-6-methoxy-7-O- $\beta$-D-glucosyl coumarin & P. spinosa $L$. & {$[30,31]$} \\
\hline 194 & 5-Hydroxy-6-methoxy-7-O- $\alpha$-D-rhamnosyl coumarin & P. spinosa $L$. & {$[30]$} \\
\hline 195 & 5,7-Dimethoxy-6-hydroxy-coumarin & P. domestica $L$. & {$[57,68]$} \\
\hline 196 & 7-Methocycoumarin & P. domestica $L$. & {$[42]$} \\
\hline 197 & Esculin & P. domestica $L$. & {$[36]$} \\
\hline 198 & Scopolin & P. domestica $L$. & [69] \\
\hline 199 & Scopoletin & P. domestica $L$. & {$[52,63,70]$} \\
\hline 200 & Magnolioside & P. domestica $L$. & {$[52,63]$} \\
\hline 201 & 6,7-Methylenedioxy-8-methoxycoumarin & P. domestica $L$. & {$[61]$} \\
\hline 202 & (3-O-cis-p-Coumaroyl- $\alpha$-D-fructofuranosyl)-(2-1)- $\alpha$-D-glucopyranoside & P. domestica $L$. & {$[69]$} \\
\hline 203 & (3-O-trans-p-Coumaroyl- $\alpha$-D-fructofuranosyl)-(2 $\longrightarrow 1)$ - $\alpha$-D-glucopyranoside & P. domestica $L$. & [69] \\
\hline 204 & 2,7-Dimethyl-2E,4E-octadienedioic acid & P. domestica $L$. & [69] \\
\hline 205 & $\alpha$-D-Glucopyranosyl 7-carboxy2-methyl-2E,4E-octadienate & P. domestica $L$. & [69] \\
\hline 206 & $\begin{array}{c}1 S \text {-(4- } \alpha \text {-D-glucopyranosyl-3-methoxyphenyl)-2R-[4-(3-hydroxypropyl)-2- } \\
\text { methoxyphenoxy]-1,3-propanediol }\end{array}$ & P. domestica $L$. & {$[69]$} \\
\hline 207 & $\alpha$-D-glucopyranosyl 9-carboxy-8-hydroxy-2,7-dimethyl-2E,4E-nonadienate & P. domestica $L$. & [69] \\
\hline 208 & $\begin{array}{l}\text { 8-Hydroxy-2,7-dimethyl-2E,4E-decadienedioic acid 1- } \alpha \text {-D-glucopyranyl ester 10- } \\
\text { Methyl ester }\end{array}$ & P. domestica $L$. & [69] \\
\hline 209 & $\alpha$-D-glucopyranosyl cinnamate & P. domestica $L$. & [69] \\
\hline 210 & (-)-Dihydrodehydrodiconiferyl alcohol & P. domestica $L$. & [70] \\
\hline 211 & $(-)$-Ficusal & P. domestica $L$. & [70] \\
\hline 212 & (E)-3, 3'-dimethoxy -4,4'-dihydroxystilbene & P. domestica $L$. & [70] \\
\hline
\end{tabular}


<smiles>CCOc1cc(-c2[o+]c3cc(O)cc(O)c3cc2OCCO)ccc1O</smiles>

$1 \mathrm{R}_{1}=\mathrm{Gal} ; \mathrm{R}_{2}=\mathrm{H}$

$2 \mathrm{R}_{1}=$ glucosyl; $\mathrm{R}_{2}=\mathrm{H}$

$3 \mathrm{R}_{1}=\alpha$-L-rhamnosyl $\beta$-D-glucose; $\mathrm{R}_{2}=\mathrm{H}$

$4 \mathrm{R}_{1}=$ acetylglucosyl; $\mathrm{R}_{2}=\mathrm{H}$

$5 \mathrm{R}_{1}=\mathrm{Xyl} ; \mathrm{R}_{2}=\mathrm{H}$

$6 \mathrm{R}_{1}=$ pentosyl; $\mathrm{R}_{2}=\mathrm{H}$

$7 \mathrm{R}_{1}=\alpha$-L-rhamnosyl $\beta$-D-glucose; $\mathrm{R}_{2}=\mathrm{CH}_{3}$

$8 \mathrm{R}_{1}=$ glucosyl; $\mathrm{R}_{2}=\mathrm{CH}_{3}$

$9 \mathrm{R}_{1}=$ acetylglucosyl; $\mathrm{R}_{2}=\mathrm{CH}_{3}$<smiles>O=C(O[C@@H]1Cc2c(O)cc(O)cc2O[C@H]1c1ccc(O)c(O)c1)c1cc(O)c(O)c(O)c1</smiles>

18

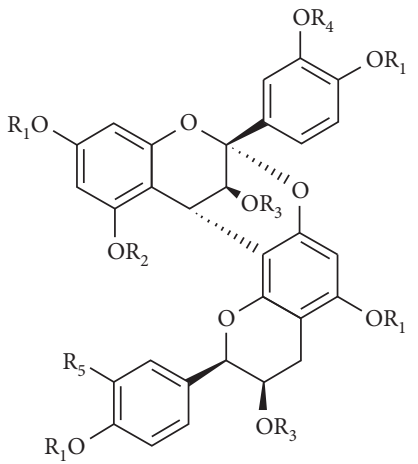

$23 \mathrm{R}_{1}=\mathrm{H} ; \mathrm{R}_{2}=\mathrm{H} ; \mathrm{R}_{3}=\mathrm{H} ; \mathrm{R}_{4}=\mathrm{H} ; \mathrm{R}_{5}=\mathrm{H}$

$24 \mathrm{R}_{1}=\mathrm{CH}_{3} ; \mathrm{R}_{2}=\mathrm{H} ; \mathrm{R}_{3}=\mathrm{H} ; \mathrm{R}_{4}=\mathrm{H} ; \mathrm{R}_{5}=\mathrm{H}$

$25 \mathrm{R}_{1}=\mathrm{H} ; \mathrm{R}_{2}=\mathrm{H} ; \mathrm{R}_{3}=\mathrm{H} ; \mathrm{R}_{4}=\mathrm{H} ; \mathrm{R}_{5}=\mathrm{OH}$

$26 \mathrm{R}_{1}=\mathrm{CH}_{3} ; \mathrm{R}_{2}=\mathrm{CH}_{3} ; \mathrm{R}_{3}=\mathrm{Ac} ; \mathrm{R}_{4}=\mathrm{CH}_{3} ; \mathrm{R}_{5}=\mathrm{OCH}_{3}$

$27 \mathrm{R}_{1}=\mathrm{CH}_{3} ; \mathrm{R}_{2}=\mathrm{Ac} ; \mathrm{R}_{3}=\mathrm{Ac} ; \mathrm{R}_{4}=\mathrm{CH}_{3} ; \mathrm{R}_{5}=\mathrm{OCH}_{3}$

$28 R_{1}=A c ; R_{2}=A c ; R_{3}=A c ; R_{4}=A c ; R_{5}=O A c$<smiles>[R3]c1cc([C@H]2Oc3cc(O)cc(O)c3CC2[R2])cc([R3])c1O</smiles>

$10 \mathrm{R}_{1}=\mathrm{H} ; \mathrm{R}_{2}=\mathrm{OH} ; \mathrm{R}_{3}=\mathrm{H} ; \mathrm{R}_{4}=\mathrm{H}$

$11 \mathrm{R}_{1}=\mathrm{OH} ; \mathrm{R}_{2}=\mathrm{H} ; \mathrm{R}_{3}=\mathrm{H} ; \mathrm{R}_{4}=\mathrm{H}$

$12 \mathrm{R}_{1}=\mathrm{H} ; \mathrm{R}_{2}=\mathrm{OH} ; \mathrm{R}_{3}=\mathrm{OH} ; \mathrm{R}_{4}=\mathrm{H}$

$13 \mathrm{R}_{1}=\mathrm{OH} ; \mathrm{R}_{2}=\mathrm{H} ; \mathrm{R}_{3}=\mathrm{OH} ; \mathrm{R}_{4}=\mathrm{H}$

$14 \mathrm{R}_{1}=\mathrm{OH} ; \mathrm{R}_{2}=\mathrm{H} ; \mathrm{R}_{3}=\mathrm{OH} ; \mathrm{R}_{4}=\mathrm{OH}$

$15 \mathrm{R}_{1}=\mathrm{H} ; \mathrm{R}_{2}=\mathrm{OH} ; \mathrm{R}_{3}=\mathrm{OH} ; \mathrm{R}_{4}=\mathrm{OH}$

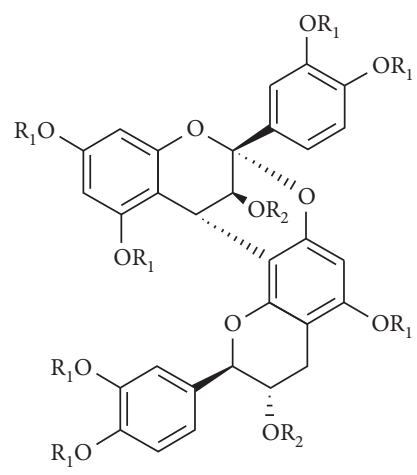

$19 \mathrm{R}_{1}=\mathrm{H} ; \mathrm{R}_{2}=\mathrm{H}$

$20 \mathrm{R}_{1}=\mathrm{CH}_{3} ; \mathrm{R}_{2}=\mathrm{Ac}$

$21 \mathrm{R}_{1}=\mathrm{Ac} ; \mathrm{R}_{2}=\mathrm{Ac}$

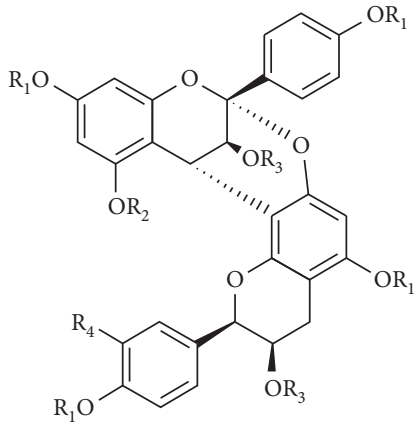

$29 \mathrm{R}_{1}=\mathrm{H} ; \mathrm{R}_{2}=\mathrm{H} ; \mathrm{R}_{3}=\mathrm{H} ; \mathrm{R}_{4}=\mathrm{OH}$ $30 \mathrm{R}_{1}=\mathrm{CH}_{3} ; \mathrm{R}_{2}=\mathrm{Ac} ; \mathrm{R}_{3}=\mathrm{Ac} ; \mathrm{R}_{4}=\mathrm{H}$

$31 \mathrm{R}_{1}=\mathrm{Ac} ; \mathrm{R}_{2}=\mathrm{Ac} ; \mathrm{R}_{3}=\mathrm{Ac} ; \mathrm{R}_{4}=\mathrm{H}$

$32 \mathrm{R}_{1}=\mathrm{CH}_{3} ; \mathrm{R}_{2}=\mathrm{Ac} ; \mathrm{R}_{3}=\mathrm{Ac} ; \mathrm{R}_{4}=\mathrm{OCH}_{3}$

$33 \mathrm{R}_{1}=\mathrm{Ac} ; \mathrm{R}_{2}=\mathrm{Ac} ; \mathrm{R}_{3}=\mathrm{Ac} ; \mathrm{R}_{4}=\mathrm{OAc}$

$34 \mathrm{R}_{1}=\mathrm{CH}_{3} ; \mathrm{R}_{2}=\mathrm{CH}_{3} ; \mathrm{R}_{3}=\mathrm{Ac} ; \mathrm{R}_{4}=\mathrm{OCH}_{3} \quad 36 \mathrm{R}=\mathrm{H}$

$35 \mathrm{R}_{1}=\mathrm{CH}_{3} ; \mathrm{R}_{2}=\mathrm{Ac} ; \mathrm{R}_{3}=\mathrm{Ac} ; \mathrm{R}_{4}=\mathrm{OCH}_{3} \quad 37 \mathrm{R}=\mathrm{OH}$

(a)

Figure 1: Continued.<smiles>[R6]C1Cc2c(O)cc(O)c(C)c2O[C@H]1c1ccc(O)c(O)c1</smiles>

$16 \mathrm{R}_{1}=\mathrm{OH} ; \mathrm{R}_{2}=\mathrm{H}$

$17 \mathrm{R}_{1}=\mathrm{H} ; \mathrm{R}_{2}=\mathrm{OH}$<smiles>C[C@@H]1[C@@H]2O[C@H]1c1c(O)cc(O)cc1O[C@]2(c1cc(O)c(O)c(O)c1)c1c(O)cc(O)c2c1O[C@H](c1ccc(O)c(O)c1)[C@@H]2O</smiles>

22

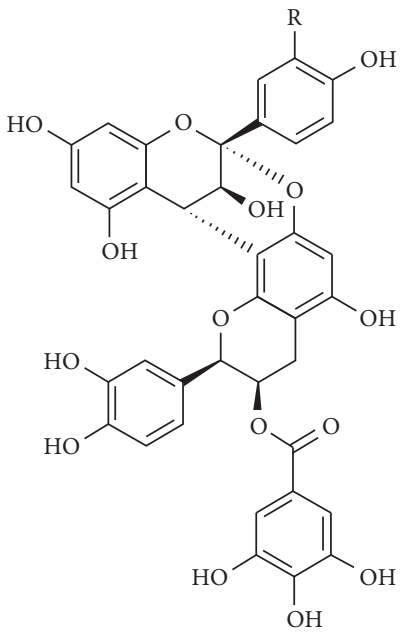




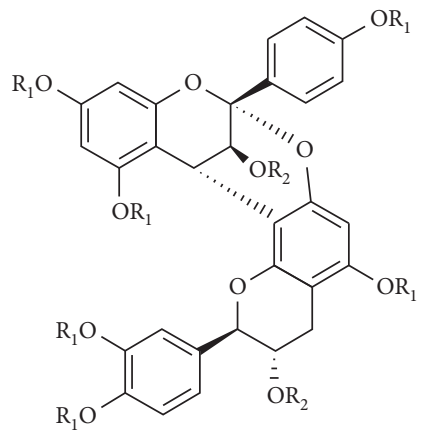

$38 \mathrm{R}_{1}=\mathrm{H} ; \mathrm{R}_{2}=\mathrm{H}$

$39 \mathrm{R}_{1}=\mathrm{Ac} ; \mathrm{R}_{2}=\mathrm{Ac}$<smiles>Oc1cc(O)c2c(c1)O[C@H](c1ccc(O)c(O)c1)[C@@H](O)[C@H]2c1c(O)cc(O)c2c1O[C@]1(c3ccc(O)c(O)c3)[C@H](O)[C@H](c3c4c(O)cc(c3O4)C[C@@H](O)[C@H](c3ccc(O)c(O)c3)O2)[C@H]1O</smiles>

42<smiles>[R]c1cc(O)c2c(=O)c([R])c(-c3ccc(O)cc3)oc2c1</smiles>

$64 \mathrm{R}_{1}=\mathrm{OH} ; \mathrm{R}_{2}=\mathrm{OH}$

$65 \mathrm{R}_{1}=\mathrm{H} ; \mathrm{R}_{2}=\mathrm{OH}$

$66 \mathrm{R}_{1}=\beta$-D-Glu; $\mathrm{R}_{2}=\mathrm{OH}$

$67 \mathrm{R}_{1}=\alpha$-L-rhamnopyranosyl; $\mathrm{R}_{2}=$ rhamnopyranosyl

$68 \mathrm{R}_{1}=\mathrm{OH} ; \mathrm{R}_{2}=\alpha$-L-rhamnopyranosyl

$69 \mathrm{R}_{1}=\alpha$-L-arabinofuranose; $\mathrm{R}_{2}=\alpha$-L-rhamnopyranosyl

$70 \mathrm{R}_{1}=\alpha$-L-arabinofuranose; $\mathrm{R}_{2}=\mathrm{OH}$

$71 \mathrm{R}_{1}$ = arabinofuranose; $\mathrm{R}_{2}=$ rhamnopyranosyl

$72 \mathrm{R}_{1}=\mathrm{O}-\beta$-D-xylopyranose; $\mathrm{R}_{2}=\alpha$-L-rhamnopyranosyl

$73 \mathrm{R}_{1}=\mathrm{O}-\beta$-D-xylopyranose; $\mathrm{R}_{2}=\mathrm{OH}$

$74 \mathrm{R}_{1}=\alpha$-L-rhamnopyranosyl; $\mathrm{R}_{2}=\mathrm{OH}$

$75 \mathrm{R}_{1}=2^{\prime \prime}$-E-p-conmaroyl- $\alpha$-L-arabinofuranose; $\mathrm{R}_{2}=\mathrm{OH}$

$76 \mathrm{R}_{1}=\alpha$-L-rhamnosyl $-\beta$-D-glucose; $\mathrm{R}_{2}=\mathrm{OH}$

$77 \mathrm{R}_{1}=\alpha$-L-arabinofuranose; $\mathrm{R}_{2}=\alpha$-L-rhamnopyranosyl

$78 \mathrm{R}_{1}=\mathrm{H} ; \mathrm{R}_{2}=\beta$-D-Glu

$79 \mathrm{R}_{1}=$ hexosyl; $\mathrm{R}_{2}=\mathrm{OH}$

$80 \mathrm{R}_{1}=$ pentosyl-rhamnopyranosyl; $\mathrm{R}_{2}=\mathrm{OH}$

$81 \mathrm{R}_{1}=$ pentosyl; $\mathrm{R}_{2}=\mathrm{OH}$

$82 \mathrm{R}_{1}=4$ " $-\beta$-D-glucopyrnosyl- $\alpha$-L-rhamnopyrnosyl; $\mathrm{R}_{2}=\mathrm{OH}$

$83 \mathrm{R}_{1}=2^{\prime \prime}$-E-p-conmaroyl- $\alpha$-L-arabinofuranose; $\mathrm{R}_{2}=\alpha$-L-rhamnopyrnosyl

$\mathrm{R}_{1}=\mathrm{OH} ; \mathrm{R}_{2}=\mathrm{OH}$

$\mathrm{R}_{1}=\alpha$-D-Glu; $\mathrm{R}_{2}=\mathrm{OH}$

$47 \mathrm{R}_{1}=\mathrm{Xyl} ; \mathrm{R}_{2}=\mathrm{OH}$

$53 \mathrm{R}_{1}=\beta$-D-Glu; $\mathrm{R}_{2}=\mathrm{OH}$

$54 \mathrm{R}_{1}=$ pentosyl; $\mathrm{R}_{2}=\mathrm{OH}$<smiles>Oc1cc(O)c2c(c1)O[C@H](c1ccc(O)c(O)c1)[C@H](O)C2c1c(O)cc(O)c2c1O[C@H](c1ccc(O)c(O)c1)[C@H](O)C2</smiles>

41<smiles>[R]c1cc(O)c2c(=O)c([R])c(-c3ccc(O)c(O)c3)oc2c1</smiles>

$44 \mathrm{R}_{1}=\mathrm{O}$-L-arabinofuranose; $\mathrm{R}_{2}=\mathrm{OH}$

$45 \mathrm{R}_{1}=\alpha$-L-rhamnosyl- $\beta$-D-glucose; $\mathrm{R}_{2}=\mathrm{OH}$

$\mathrm{R}_{1}=$ arabinoside; $\mathrm{R}_{2}=\mathrm{OH}$

$\mathrm{R}_{1}=\alpha$-L-rhamnopyranosyl; $\mathrm{R}_{2}=\mathrm{OH}$

$50 \mathrm{R}_{1}=\mathrm{OH} ; \mathrm{R}_{2}=\alpha$-L-rhamnopyranosyl

$\mathrm{R}_{1}=O-\beta-\mathrm{D}-\mathrm{Gal} ; \mathrm{R}_{2}=\mathrm{OH}$

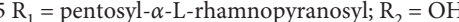

$56 \mathrm{R}_{1}=$ pentosylhexoside; $\mathrm{R}_{2}=\mathrm{OH}$

$\mathrm{R}_{1}=$ pentosyl-pentosyl; $\mathrm{R}_{2}=\mathrm{OH}$

践 = acetylhexoside; $\mathrm{R}_{2}=\mathrm{OH}$

60

$=4 "$ - $\beta$-D-glucopyrnosyl- $\alpha$-L-rhamnopyrnosyl; $\mathrm{R}_{2}=\mathrm{OH}$

年

$62 \mathrm{R}_{1}=2^{\prime \prime}$ - $\beta$-D-glucopyrnosyl $\alpha$-L-arabinofuranose; $\mathrm{R}_{2}=\mathrm{OH}$

$3 \mathrm{R}_{1}=\beta$-D-galactopyranosyloxy; $\mathrm{R}_{2}=\mathrm{OH}$<smiles>[R]c1cc(-c2oc3c([R])c(O)cc(O)c3c(=O)c2[R])cc([R3])c1[R]</smiles>

$84 \mathrm{R}_{1}=\mathrm{OH} ; \mathrm{R}_{2}=\mathrm{H} ; \mathrm{R}_{3}=\mathrm{OH} ; \mathrm{R}_{4}=\mathrm{OH} ; \mathrm{R}_{5}=\mathrm{OH}$ $85 \mathrm{R}_{1}=\mathrm{H} ; \mathrm{R}_{2}=\mathrm{H} ; \mathrm{R}_{3}=\mathrm{OH} ; \mathrm{R}_{4}=\mathrm{OH} ; \mathrm{R}_{5}=\mathrm{H}$ $86 \mathrm{R}_{1}=\mathrm{H} ; \mathrm{R}_{2}=\mathrm{H} ; \mathrm{R}_{3}=\mathrm{OH} ; \mathrm{R}_{4}=$ b-D-Glu; $\mathrm{R}_{5}=\mathrm{H}$ $87 \mathrm{R}_{1}=\mathrm{OH} ; \mathrm{R}_{2}=\mathrm{OCH}_{3} ; \mathrm{R}_{3}=\mathrm{H} ; \mathrm{R}_{4}=\mathrm{OCH}_{3} ; \mathrm{R}_{5}=\mathrm{H}$ $88 \mathrm{R}_{1}=\mathrm{OH} ; \mathrm{R}_{2}=\mathrm{H} ; \mathrm{R}_{3}=\mathrm{H} ; \mathrm{R}_{4}=\mathrm{OCH}_{3} ; \mathrm{R}_{5}=\mathrm{H}$ $89 \mathrm{R}_{1}=O-\alpha-\mathrm{L}-\mathrm{Rha}-\beta-\mathrm{D}-\mathrm{Glu} ; \mathrm{R}_{2}=\mathrm{H} ; \mathrm{R}_{3}=\mathrm{OCH}_{3}$; $\mathrm{R}_{4}=\mathrm{OH} ; \mathrm{R}_{5}=\mathrm{H}$

$90 \mathrm{R}_{1}=\mathrm{O}$-Glu; $\mathrm{R}_{2}=\mathrm{H} ; \mathrm{R}_{3}=\mathrm{OCH}_{3} ; \mathrm{R}_{4}=\mathrm{OH} ; \mathrm{R}_{5}=\mathrm{H}$ $91 \mathrm{R}_{1}=O$-Gal; $\mathrm{R}_{2}=\mathrm{H} ; \mathrm{R}_{3}=\mathrm{OCH}_{3} ; \mathrm{R}_{4}=\mathrm{OH} ; \mathrm{R}_{5}=\mathrm{H}$ $92 \mathrm{R}_{1}=\mathrm{OH} ; \mathrm{R}_{2}=\mathrm{H} ; \mathrm{R}_{3}=\mathrm{OH} ; \mathrm{R}_{4}=\beta$-D-Glu; $\mathrm{R}_{5}=\mathrm{H}$<smiles>[R]c1ccc(C2Oc3c([R3])c(O)c([R])c(O)c3C(=O)C2[R])cc1[R]</smiles>

$93 \mathrm{R}_{1}=\mathrm{H} ; \mathrm{R}_{2}=\mathrm{H} ; \mathrm{R}_{3}=\mathrm{H} ; \mathrm{R}_{4}=\mathrm{OH} ; \mathrm{R}_{5}=\mathrm{OCH}_{3}$ $94 \mathrm{R}_{1}=\mathrm{H} ; \mathrm{R}_{2}=\mathrm{H} ; \mathrm{R}_{3}=\mathrm{H} ; \mathrm{R}_{4}=\mathrm{H} ; \mathrm{R}_{5}=\mathrm{OCH}_{3}$ $95 \mathrm{R}_{1}=\mathrm{OH} ; \mathrm{R}_{2}=\mathrm{H} ; \mathrm{R}_{3}=\mathrm{H} ; \mathrm{R}_{4}=\mathrm{H} ; \mathrm{R}_{5}=\mathrm{OCH}_{3}$ $96 \mathrm{R}_{1}=\mathrm{H} ; \mathrm{R}_{2}=\mathrm{H} ; \mathrm{R}_{3}=\mathrm{H} ; \mathrm{R}_{4}=\mathrm{H} ; \mathrm{R}_{5}=\mathrm{OH}$ $97 \mathrm{R}_{1}=\mathrm{OH} ; \mathrm{R}_{2}=\mathrm{H} ; \mathrm{R}_{3}=\mathrm{OCH}_{3} ; \mathrm{R}_{4}=\mathrm{H} ; \mathrm{R}_{5}=\mathrm{OCH}_{3}$ $98 \mathrm{R}_{1}=\mathrm{OH} ; \mathrm{R}_{2}=\mathrm{OCH}_{3} ; \mathrm{R}_{3}=\mathrm{H} ; \mathrm{R}_{4}=\mathrm{H} ; \mathrm{R}_{5}=\mathrm{OCH}_{3}$ $99 \mathrm{R}_{1}=\mathrm{OCH}_{3} ; \mathrm{R}_{2}=\mathrm{H} ; \mathrm{R}_{3}=\mathrm{H} ; \mathrm{R}_{4}=\mathrm{H} ; \mathrm{R}_{5}=\mathrm{OH}$ $100 \mathrm{R}_{1}=\mathrm{OCH}_{3} ; \mathrm{R}_{2}=\mathrm{H} ; \mathrm{R}_{3}=\mathrm{H} ; \mathrm{R}_{4}=\mathrm{H} ; \mathrm{R}_{5}=\mathrm{OCH}_{3}$ $101 \mathrm{R}_{1}=\mathrm{H} ; \mathrm{R}_{2}=\mathrm{H} ; \mathrm{R}_{3}=\mathrm{H} ; \mathrm{R}_{4}=\mathrm{H} ; \mathrm{R}_{5}=\mathrm{OCH}_{3}$ $102 \mathrm{R}_{1}=\mathrm{H} ; \mathrm{R}_{2}=\mathrm{H} ; \mathrm{R}_{3}=\mathrm{OCH}_{3} ; \mathrm{R}_{4}=\mathrm{H} ; \mathrm{R}_{5}=\mathrm{OCH}_{3}$ $103 \mathrm{R}_{1}=\mathrm{O}-\mathrm{a}-\mathrm{L}-\mathrm{Rh}$; $\mathrm{R}_{2}=\mathrm{H} ; \mathrm{R}_{3}=\mathrm{H} ; \mathrm{R}_{4}=\mathrm{H} ; \mathrm{R}_{5}=\mathrm{OH}$ $104 \mathrm{R}_{1}=\mathrm{O}-\beta$-D-Glu; $\mathrm{R}_{2}=\mathrm{H} ; \mathrm{R}_{3}=\mathrm{H} ; \mathrm{R}_{4}=\mathrm{H} ; \mathrm{R}_{5}=\mathrm{OH}$

(b)

Figure 1: Continued. 
<smiles>[R20]c1cc(OC)c2c(=O)cc(-c3ccc(COC(=O)CC(CC)CCCC)cc3)oc2c1[R]</smiles><smiles></smiles>

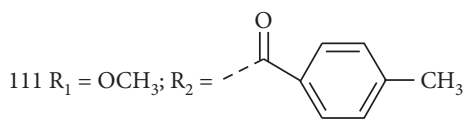<smiles>O=C(CCc1ccc(O)cc1)c1c(O)cc(O)cc1OC(O)C(O)C(O)CO</smiles>

112<smiles>COc1cc(O)c2c(=O)cc(-c3cc(OC)c(O)cc3O)oc2c1</smiles>

113<smiles>[R2]Oc1c([R3])cc(-c2cc(=O)c3c([R])c([R])c(OC)cc3o2)c(O)c1[R3]</smiles><smiles>[R][R]OC</smiles>

$\mathrm{R}_{3}=\mathrm{OH}$

$\mathrm{R}_{3}=\mathrm{OCH}_{3}$

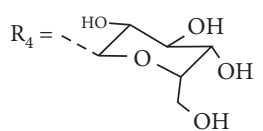

$\mathrm{R}_{5}=\mathrm{OH}$<smiles>[R]C[C@H]1O[C@@H](CO)[C@@H](CO)[C@H](O)[C@@H]1O</smiles>

$\mathrm{R}_{5}=\mathrm{OCH}_{3}$

105

106<smiles>[R]OC(=O)/C=C/c1c(O)cc2oc(C)c(Cc3ccc(OC4OC(O)C(O)C(O)C4O)c(OC)c3)c(=O)c2c1OC</smiles>

$107 \mathrm{R}=\mathrm{H}$

$108 \mathrm{R}=\mathrm{CH}_{2} \mathrm{CH}_{2} \mathrm{CH}_{3}$

$109 \mathrm{R}=$ hydroxyphenyl

(c)

Figure 1: Chemical structures of flavonoids from Prunus species.

all of which show high antioxidant activity [50, 75]. Excessive presence of various natural and edible pigments in leaves and branches of $P$. cerasifera make it more demanding in the world market, especially the anthocyanins rich in red edible pigments for beverage industry [29, 76-78].

3.2. Prunus domestica. The Cherry plum (Prunus domestica L., Rosaceae) is famous as "Mirabelle plum" or "Myrobalan," and its fruits display a huge variation in size, shape, taste, and appearance [24]. Fresh fruits of $P$. domestica are processed into dried functional food so that they keep its potential health effects shown by the presence of plenty of phenolics and antioxidants [54]. Prunus kernels which actually are the dried form of the fruits are achieved at $85-90^{\circ} \mathrm{C}$ for 18 hours [79] and have been utilized in medicine for centuries [63]. In different parts of the world, especially in Southeast Asia, prunes are being administered alone or in combination with other medicines to cure menstrual disorders, leucorrhoea, and debility after the miscarriage [80]. Moreover, plums have a laxative effect due to high fibre contents and higher phenolic contents $[81,82]$. Certain studies reported that high phenolics may pose positive health effects on the development and strengthening of bones and memory-related issues, reduce inflammations, release constipation, and scavenge ROS [83-87]. The higher antioxidant activity of extracts of $P$. domestica is mainly because of the presence of phenolic compounds, especially the isomers of caffeoylquinic acid $[6,63]$.

The oral administration of the Prunus fruit extracts (75, $100,150 \mathrm{mg} / \mathrm{kg}$ ) to male mice gave much higher learning and memory enhancement [88]. The chlorogenic acid isolated from $P$. domestica reduces the anxiolytic-like effect in vitro which is linked with anxiety behaviour and provides protection to granulocytes by avoiding ROS efflux [64]. The improvement of bone structure and its biomechanical properties is linked with the usage of higher doses of plums that downregulates the expression of TNF- $\alpha$ in lymphocytes [89], as well as retention of bone calcium ions [86].

The fruit flesh extract of $P$. domestica inhibited the cell proliferation of breast cancer cells (MCF-7, MDA-MB-453, and MCF-10A cell lines), as well as reduces the toxicity levels in the normal cells [84]. Furthermore, prunes are also found to reduce various other cancers such as colon cancer by inducing apoptosis without any harm to the normal neighbouring cells [90-92]. For human liver cancer cells 
<smiles>[R]c1c(Br)cc(C(=O)O)cc1Br</smiles>

$114 \mathrm{R}_{1}=\mathrm{OCH}_{3} ; \mathrm{R}_{2}=\mathrm{OH} ; \mathrm{R}_{3}=\mathrm{H}$

$115 \mathrm{R}_{1}=\mathrm{OH} ; \mathrm{R}_{2}=\mathrm{OH} ; \mathrm{R}_{3}=\mathrm{H}$

$116 \mathrm{R}_{1}=\mathrm{H} ; \mathrm{R}_{2}=\mathrm{OH} ; \mathrm{R}_{3}=\mathrm{H}$

$117 \mathrm{R}_{1}=\mathrm{OCH}_{3} ; \mathrm{R}_{2}=O-\beta-\mathrm{D}-\mathrm{glc} ; \mathrm{R}_{3}=\mathrm{H}$

$118 \mathrm{R}_{1}=\mathrm{OCH} 3 ; \mathrm{R}_{2}=\mathrm{O}-\beta$-D-glucosyl; $\mathrm{R}_{3}=\mathrm{H}$

$119 \mathrm{R}_{1}=\mathrm{OH} ; \mathrm{R}_{2}=\mathrm{OH} ; \mathrm{R}_{3}=\mathrm{OH}$

$120 \mathrm{R}_{1}=\mathrm{OCH}_{3} ; \mathrm{R}_{2}=\mathrm{OH} ; \mathrm{R}_{3}=\mathrm{OCH}_{3}$

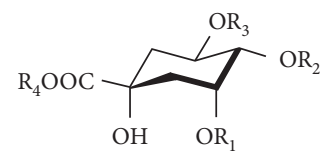

$128 \mathrm{R}_{1}=$ caffeoyl; $\mathrm{R}_{2}=\mathrm{H} ; \mathrm{R}_{3}=\mathrm{H} ; \mathrm{R}_{4}=\mathrm{H}$

$129 \mathrm{R}_{1}=\mathrm{H} ; \mathrm{R}_{2}=$ caffeoyl; $\mathrm{R}_{3}=\mathrm{H} ; \mathrm{R}_{4}=\mathrm{H}$

$130 \mathrm{R}_{1}=\mathrm{H} ; \mathrm{R}_{2}=\mathrm{H} ; \mathrm{R}_{3}=$ caffeoyl; $\mathrm{R}_{4}=\mathrm{H}$

$131 \mathrm{R}_{1}=$ caffeoyl; $\mathrm{R}_{2}=\mathrm{H} ; \mathrm{R}_{3}=\mathrm{H} ; \mathrm{R}_{4}=\mathrm{CH}_{3}$

$132 \mathrm{R}_{1}=\mathrm{H} ; \mathrm{R}_{2}=$ caffeoyl; $\mathrm{R}_{3}=\mathrm{H} ; \mathrm{R}_{4}=\mathrm{CH}_{3}$

$133 \mathrm{R}_{1}=\mathrm{H} ; \mathrm{R}_{2}=\mathrm{H} ; \mathrm{R}_{3}=$ caffeoyl; $\mathrm{R}_{4}=\mathrm{CH}_{3}$

$134 \mathrm{R}_{1}=$ feruloyl; $\mathrm{R}_{2}=\mathrm{H} ; \mathrm{R}_{3}=\mathrm{H} ; \mathrm{R}_{4}=\mathrm{H}$

$135 \mathrm{R}_{1}=\mathrm{H} ; \mathrm{R}_{2}=$ feruloyl; $\mathrm{R}_{3}=\mathrm{H} ; \mathrm{R}_{4}=\mathrm{H}$

$136 \mathrm{R}_{1}=$ comaroyl; $\mathrm{R}_{2}=\mathrm{H} ; \mathrm{R}_{3}=\mathrm{H} ; \mathrm{R}_{4}=\mathrm{H}$

$137 \mathrm{R}_{1}=\mathrm{H} ; \mathrm{R}_{2}=$ comaroyl; $\mathrm{R}_{3}=\mathrm{H} ; \mathrm{R}_{4}=\mathrm{H}$

$138 \mathrm{R}_{1}=\mathrm{H} ; \mathrm{R}_{2}=\mathrm{H} ; \mathrm{R}_{3}=$ comaroyl; $\mathrm{R}_{4}=\mathrm{H}$

$139 \mathrm{R}_{1}=$ comaroyl; $\mathrm{R}_{2}=\mathrm{H} ; \mathrm{R}_{3}=\mathrm{H} ; \mathrm{R}_{4}=\mathrm{CH}_{3}$<smiles>[R3]C=Cc1ccc([R8])c([R])c1</smiles>

$121 \mathrm{R}_{1}=\mathrm{OH} ; \mathrm{R}_{2}=\mathrm{OH} ; \mathrm{R}_{3}=\mathrm{COOH}$

$122 \mathrm{R}_{1}=\mathrm{OH} ; \mathrm{R}_{2}=\mathrm{OH} ; \mathrm{R}_{3}=\mathrm{COOCH}_{3}$

$123 \mathrm{R}_{1}=\mathrm{OCH}_{3} ; \mathrm{R}_{2}=\mathrm{OH} ; \mathrm{R}_{3}=\mathrm{COOH}$

$124 \mathrm{R}_{1}=\mathrm{H} ; \mathrm{R}_{2}=\mathrm{OH} ; \mathrm{R}_{3}=\mathrm{COOH}$

$125 \mathrm{R}_{1}=\mathrm{H} ; \mathrm{R}_{2}=\mathrm{H} ; \mathrm{R}_{3}=\mathrm{COOH}$

$126 \mathrm{R}_{1}=\mathrm{OCH}_{3} ; \mathrm{R}_{2}=O$ - $\beta$-D-glucosyl; $\mathrm{R}_{3}=\mathrm{COOH}$

$127 \mathrm{R}_{1}=\mathrm{OCH} 3 ; \mathrm{R}_{2}=O$ - $\beta$-D-glucosyl; $\mathrm{R}_{3}=\mathrm{CH}_{2} \mathrm{OH}$<smiles>CCc1ccc(O)c(O)c1</smiles>

$\mathrm{OH}$

144<smiles>O=c1oc2c(O)c(O)cc3c(=O)oc4c(O)c(O)cc1c4c23</smiles>

145<smiles>[R]OC(=O)/C=C/c1ccc(O)c([R])c1</smiles>

$148 \mathrm{R}_{1}=$ hexoside; $\mathrm{R}_{2}=\mathrm{OH}$

$149 \mathrm{R}_{1}=$ hexoside; $\mathrm{R}_{2}=\mathrm{H}$<smiles>[R3]c1cccc(C(=O)O)c1[R]</smiles>

$140 \mathrm{R}_{1}=\mathrm{OH} ; \mathrm{R}_{2}=\mathrm{H}$

$141 \mathrm{R}_{1}=\mathrm{CH}_{3} ; \mathrm{R}_{2}=\mathrm{CH}_{3}$<smiles>O=C(O)C1C=C(O)C(O)C(O)C1</smiles>

$142 \mathrm{R}=\mathrm{H}$

$143 \mathrm{R}=$ caffeoyl<smiles>[R]c1cc(CCC(=O)O)ccc1O</smiles>

$146 \mathrm{R}=\mathrm{H}$

$147 \mathrm{R}=\mathrm{OH}$<smiles>O=C(/C=C/c1ccc(O)c(O)c1)OC(Cc1ccc(O)c(O)c1)C(=O)O</smiles>

151<smiles>CC1=CC(=O)CC(C)(C)C1/C=C/C(C)=C\C(=O)O</smiles>

$152 \mathrm{R}=\mathrm{H}$

$153 \mathrm{R}=\beta$-D-glucosyl<smiles>C[C@H]1[C@@H](CO)O[C@@H](OC(=O)c2ccc(O)c(O)c2)[C@H](O)[C@@H]1O</smiles><smiles>C/C(C=C[C@]12OO[C@@](C)(C[C@@H](O)[C@H]1O)C2(C)C)=C/C(=O)O</smiles>

154

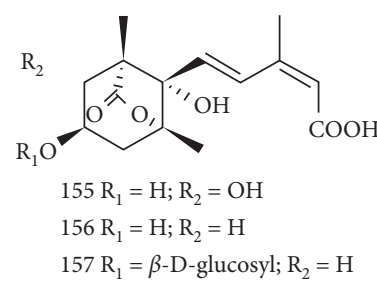<smiles>CC1=CC(=O)CC(C)(C)C1(O)/C=C/[C@H](C)O[C@@H]1O[C@H](CO)[C@@H](O)[C@H](O)[C@H]1O</smiles>

Figure 2: Chemical structures of phenolic acids from Prunus species.

(HepG2), prunes aid in atntiproliferation activity [90]. Moreover, the polyphenolics in prunes help in reduction in inflammatory markers such as Cyclooxygenase 2 (COX-2) [92]. The fruit extract of dried plums have been reported to be rich in polyphenolic compounds and showed a huge reduction in the inflammatory markers such as nitric oxide and melondialdehyde production in a dose-dependent manner [93]. On the other hand, another class of phytocompounds reported in $P$. domestica is polysaccharides, which reduces and completely inhibited gastric lesions in a rat model [94].

The fruit extracts of $P$. domestica showed positive results against peptic ulcer in Wistar albino rats, and it was said that due to the presence of higher amounts of polyphenolics in the fruit juices of plumes, it showed strong antioxidant potential which reduces the oxidative stress and engages various acids to neutralize the corrosive effects of various acids and, hence, appeared as antiulcerogenic [95]. Phenolic 
<smiles>[R9]c1cc(Br)cc(Br)c1Br</smiles>

$159 \mathrm{R}_{1}=\mathrm{CHO} ; \mathrm{R}_{2}=\mathrm{H} ; \mathrm{R}_{3}=\mathrm{OH} ; \mathrm{R}_{4}=\mathrm{H}$

$160 \mathrm{R}_{1}=\mathrm{COOH} ; \mathrm{R}_{2}=\mathrm{H} ; \mathrm{R}_{3}=\mathrm{H} ; \mathrm{R}_{4}=\mathrm{H}$

$161 \mathrm{R}_{1}=\mathrm{CHO} ; \mathrm{R}_{2}=\mathrm{OCH}_{3} ; \mathrm{R}_{3}=\mathrm{OH} ; \mathrm{R}_{4}=\mathrm{OCH}_{3}$

$162 \mathrm{R}_{1}=\mathrm{OCH}_{3} ; \mathrm{R}_{2}=\mathrm{OH} ; \mathrm{R}_{3}=\mathrm{CHO} ; \mathrm{R}_{4}=\mathrm{H}$

$163 \mathrm{R}_{1}=\mathrm{CHO} ; \mathrm{R}_{2}=\mathrm{OCH}_{3} ; \mathrm{R}_{3}=\mathrm{OH} ; \mathrm{R}_{4}=\mathrm{H}$

$164 \mathrm{R}_{1}=\mathrm{CHO} ; \mathrm{R}_{2}=\mathrm{OH} ; \mathrm{R}_{3}=\mathrm{OH} ; \mathrm{R}_{4}=\mathrm{H}$

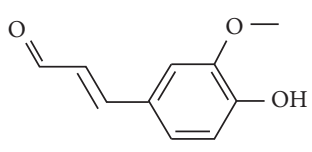

170<smiles>[R]c1cc(/C=C/C=O)cc(Br)c1O</smiles>

$165 \mathrm{R}_{1}=\mathrm{H} ; \mathrm{R}_{2}=\mathrm{OCH}_{3}$ $166 \mathrm{R}_{1}=\mathrm{OCH}_{3} ; \mathrm{R}_{2}=\mathrm{OCH}_{3}$

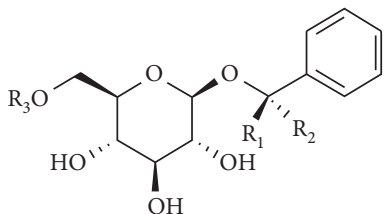

$167 \mathrm{R}_{1}=\mathrm{CN} ; \mathrm{R}_{2}=\mathrm{H} ; \mathrm{R}_{3}=\beta$-D-glucosyl $168 \mathrm{R}_{1}=\mathrm{CN} ; \mathrm{R}_{2}=\mathrm{H} ; \mathrm{R}_{3}=\mathrm{H}$ $169 \mathrm{R}_{1}=\mathrm{H} ; \mathrm{R}_{2}=\mathrm{CN} ; \mathrm{R}_{3}=\mathrm{H}$<smiles>COc1cc([C@@H]2OC[C@H]3[C@H]2CO[C@H]3c2ccc(O[C@@H]3OC(CO)[C@@H](O)[C@H](O)[C@H]3O)c(OC)c2)ccc1O</smiles>

171

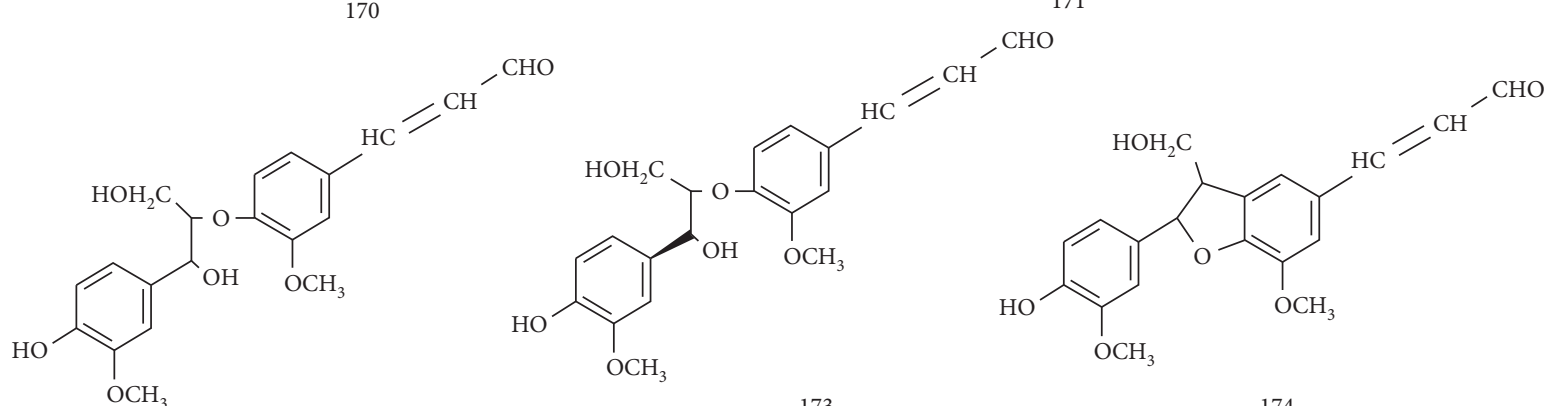

172

173

174<smiles>O=C(O)C=CC(=O)O</smiles>

175<smiles>[R]C(CC(=O)O)C(=O)O</smiles>

$176 \mathrm{R}=\mathrm{OH}$ $177 \mathrm{R}=\mathrm{H}$<smiles>[R]C1CC[C@]2(C)C(=CC[C@@H]3[C@@H]4CC[C@H]([C@H](C)CC[C@@H](CC)C(C)C)[C@]4(C)CC[C@H]32)C1</smiles>

$185 \mathrm{R}=\mathrm{OH}$ $186 \mathrm{R}=\beta$-D-glucosyl<smiles>O=C(O)CC(CC(=O)O)C(=O)O</smiles>

178<smiles>O=Cc1ccc(CO)n1C1CC(=O)NC1=O</smiles><smiles>O=Cc1ccc(CO)o1</smiles>

181

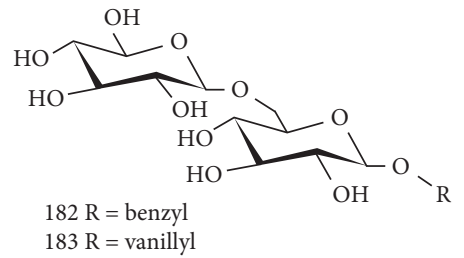

184

179

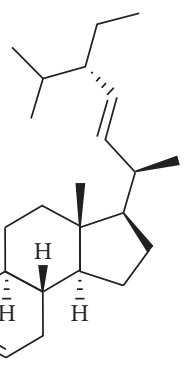

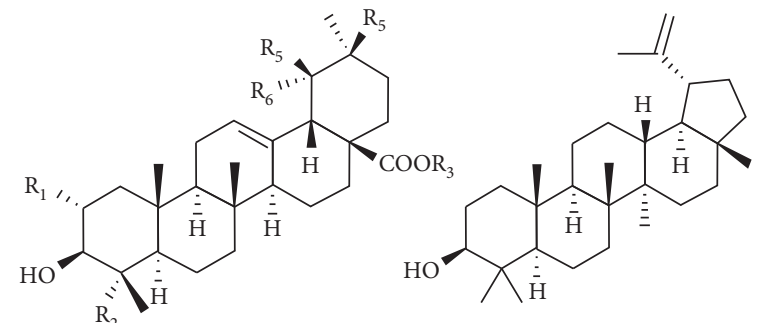

191

$188 \mathrm{R}_{1}=\mathrm{H} ; \mathrm{R}_{2}=\mathrm{CH}_{3} ; \mathrm{R}_{3}=\mathrm{H} ; \mathrm{R}_{4}=\mathrm{H} ; \mathrm{R}_{5}=\mathrm{CH}_{3} ; \mathrm{R}_{6}=\mathrm{H}$

$189 \mathrm{R}_{1}=\mathrm{OH} ; \mathrm{R}_{2}=\mathrm{CH}_{2} \mathrm{OH} ; \mathrm{R}_{3}=\mathrm{H} ; \mathrm{R}_{4}=\mathrm{CH}_{3} ; \mathrm{R}_{5}=\mathrm{H} ; \mathrm{R}_{6}=\mathrm{H}$

$190 \mathrm{R}_{1}=\mathrm{OH} ; \mathrm{R}_{2}=\mathrm{CH}_{2} \mathrm{OH} ; \mathrm{R}_{3}=\beta$-D-Glu; $\mathrm{R}_{4}=\mathrm{H} ; \mathrm{R}_{5}=\mathrm{CH}_{3} ; \mathrm{R}_{6}=\mathrm{OH}$

(a)

Figure 3: Continued. 


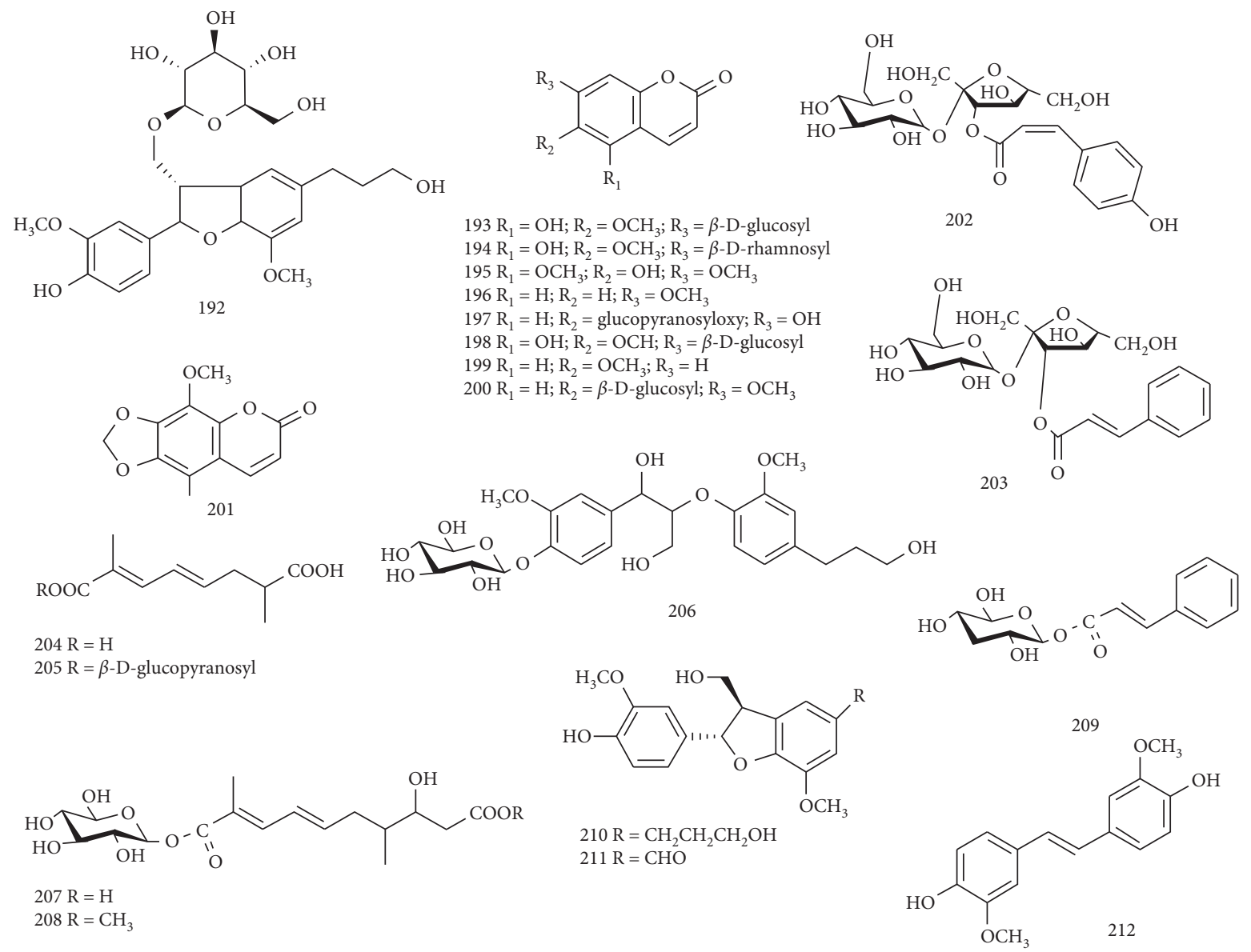

(b)

Figure 3: Chemical structures of other compounds from Prunus species.

compounds reported in $P$. domestica minimized the $\mathrm{H}_{2} \mathrm{O}_{2}$ induced oxidative stress through reducing the intracellular ROS accumulation in granulocytes [96]. Prunes are stated as highly useful to protect against cardiovascular disorders to regulate or modulate blood pressure, prevent atherosclerosis, and boost high-density lipoproteins (HDL) [97]. P. domestica fruit juice (juice concentrate + prune puree + water $+7 \%$ fructose) has laxative effects on bowel functions in individuals suffering gastrointestinal issues and increases flatulence [98]. In another study, $P$. domestica fruit extracts were studied for their antiallergic responses, and it was reported that it reduces the type-I allergic symptoms in mice by adjusting type- 1 helper T-cell/type- 2 helper T-cell balance and suppression of mast cell degranulation [99].

3.3. Prunus salicina Lindl. The oriental plum (Prunus salicina Lindley, Rosaceae), locally called as Chinese plum, is reported to be a rich source of various pigments such as anthocyanins and polyphenolic compounds. Foods enriched with polyphenolic compounds using oriental plum can improve the symptoms of neurodegenerative diseases by reducing the brain cholesterol levels and upregulation of neurodegenerative-related proteins [100]. The crude extracts of the peels and flesh of $P$. salicina fruit prepared in acetone have strong anticancer properties as reported for inducing apoptosis in MDA-MB-231 cells [53, 101]. Moreover, $P$. salicina fruits are rich in phenolic antioxidants $(82 \%)$, of which a nitrogenous compound amygdalin is in higher quantity, which is banned by the FDA as a cancer chemotherapeutic agent [53].

The $P$. salicina fruits extracts were prepared at various levels of fruit maturity, i.e., immature, partial-mature, and fully mature fruits, and these extracts showed strong anticancer potential against various cancer cell lines, viz. HepG2, Kato11, Hela, U937 lukaemia cells, MCF-7 cells, and MDAMB-231 human breast cancer cell lines [101]. The mechanism for this anticancer activity was due to the cytotoxic effects of the $P$. salicina fruit extracts containing polyphenolics that activate apoptotic pathway leading to the programmed cell death [101]. In another study, $P$. salicina fruit juices showed antiadipogenic effects and reduce inflammations, blood glucose levels, triglycerides, and high-density lipoproteins (HDL) cholesterol levels in obese rats [102]. The 
fruit extracts of $P$. salicina are rich bioactive compounds when mixed with the food supplements which help reduce mite allergic responses [99].

3.4. Prunus spinosa L. Blackthorn or sloe (Prunus spinosa L., Rosaceae) is widely cultivated throughout the world [103]. P. spinosa is resistant to cold, drought, and calcareous soils and represents one of the ancestors of $P$. domestica [104]. Like all other Chinese prunes, $P$. spinosa also bears strong biological constituents. Active components of the plant are believed to be polyphenols, including flavonoids and A-type proanthocyanidins, anthocyanins, coumarins, and phenolic acids, forming unique and diversified profiles in particular organs, among which the flowers are the least characterized [30, 33, 43, 47, 105-108]. The unique composition of phytochemicals in the $P$. spinosa plant may correspond to the distinctive activity profile reported by traditional Chinese medication systems. Other than traditional Chinese medicine, huge literature reports ethnomedicinal and ethnopharmacological uses of $P$. spinosa, which showed its potential benefits to cure various diseases $[1,32]$. $P$. spinosa fruits and its juice could be considered as a valuable source of antioxidant compounds for nutritional supplementation, as well as of herbal medicine [18]. P. spinosa L. flowers are a traditional herbal medicine recommended for the adjunctive treatment of oxidative stress-related diseases [65].

\section{Conclusions and Further Prospective}

The major phytochemicals have been isolated from four Chinese plum species including polyphenols, flavonoids, and anthocyanins. There occur huge variations in the total phytochemicals contents in different species, which make these different species to have different biological activities in multiple disease conditions, and even the same variety growing under different edaphic conditions may have different antioxidant capacities. Moreover, 212 known compounds have been reported to be present in these four Chinese plum species, which are helpful to evade chronic oxidative stress-mediated diseases. Moreover, it is suggested to perform some extensive and in-depth studies to find new phytochemicals from these four Chinese plum species which could boost the local industry to fulfill the increasing demands.

\section{Data Availability}

No data were used to support this study.

\section{Conflicts of Interest}

The authors declare that there are no conflicts of interest regarding the publication of this paper.

\section{Acknowledgments}

This research was funded by the Key Lab of Natural Product Chemistry and Application at Universities of Education
Department of Xinjiang Uygur Autonomous Region (2019YSHXZD02).

\section{References}

[1] V. Poonam, Raunak, G. Kumar et al., "Chemical constituents of the genus prunus and their medicinal properties," Current Medicinal Chemistry, vol. 18, no. 25, pp. 3758-3824, 2011.

[2] E. O. Igwe and K. E. Charlton, "A systematic review on the health effects of plums (Prunus domestica and Prunus salicina)," Phytotherapy Research, vol. 30, no. 5, pp. 701-731, 2016.

[3] J. Fang, "Apigenin inhibits tumor angiogenesis through decreasing HIF- $1 \alpha$ and VEGF expression," Carcinogenesis, vol. 28, no. 4, pp. 858-864, 2007.

[4] Q. Zhou, B. Yan, X. Hu, X.-B. Li, J. Zhang, and J. Fang, "Luteolin inhibits invasion of prostate cancer PC3 cells through E-cadherin," Molecular Cancer Therapeutics, vol. 8, no. 6, pp. 1684-1691, 2009.

[5] B. Fu, J. Xue, Z. Li, X. Shi, B.-H. Jiang, and J. Fang, "Chrysin inhibits expression of hypoxia-inducible factor-1 through reducing hypoxia-inducible factor-1 stability and inhibiting its protein synthesis," Molecular Cancer Therapeutics, vol. 6, no. 1, pp. 220-226, 2007.

[6] S.-i. Kayano, H. Kikuzaki, N. Fukutsuka, T. Mitani, and N. Nakatani, "Antioxidant activity of prune (Prunus domestica L.) constituents and a new synergist," Journal of Agricultural and Food Chemistry, vol. 50, no. 13, pp. 37083712, 2002.

[7] H. J. Kim, M.-H. Yu, and I.-S. Lee, "Inhibitory effects of methanol extract of plum (Prunus salicina L., cv. 'Soldam') fruits against benzo $(\alpha)$ pyrene-induced toxicity in mice," Food and Chemical Toxicology, vol. 46, no. 11, pp. 3407-3413, 2008.

[8] J. L. Donovan, A. S. Meyer, and A. L. Waterhouse, "Phenolic composition and antioxidant activity of prunes and prune juice (Prunus domestica)," Journal of Agricultural and Food Chemistry, vol. 46, no. 4, pp. 1247-1252, 1998.

[9] N. Pellegrini, M. Serafini, S. Salvatore, D. Del Rio, M. Bianchi, and F. Brighenti, "Total antioxidant capacity of spices, dried fruits, nuts, pulses, cereals and sweets consumed in Italy assessed by three different in vitro assays," Molecular Nutrition \& Food Research, vol. 50, no. 11, pp. 1030-1038, 2006.

[10] W. Dröge, "Free radicals in the physiological control of cell function," Physiological Reviews, vol. 82, no. 1, pp. 47-95, 2002.

[11] E. Niki, "Oxidative stress and antioxidants: distress or eustress?" Archives of Biochemistry and Biophysics, vol. 595, pp. 19-24, 2016.

[12] Y. Peng, R. Gan, H. Li et al., "Absorption, metabolism, and bioactivity of vitexin: recent advances in understanding the efficacy of an important nutraceutical," Critical Reviews in Food Science and Nutrition, pp. 1-16, 2020.

[13] Y. Wang, X. Chen, Y. Zhang, and X. Chen, "Antioxidant activities and major anthocyanins of myrobalan plum (Prunus cerasifera Ehrh.)," Journal of Food Science, vol. 77, no. 4, pp. C388-C393, 2012.

[14] F.-F. Chen, J. Sang, Y. Zhang, and J. Sang, "Development of a green two-dimensional HPLC-DAD/ESI-MS method for the determination of anthocyanins from Prunus cerasifera var. atropurpurea leaf and improvement of their stability in energy drinks," International Journal of Food Science \& Technology, vol. 53, no. 6, pp. 1494-1502, 2018. 
[15] A. Venter, E. Joubert, and D. de Beer, "Characterisation of phenolic compounds in South African plum fruits (Prunus salicina Lindl.) using HPLC coupled with diode-array, fluorescence, mass spectrometry and on-Line antioxidant detection," Molecules, vol. 18, no. 5, pp. 5072-5090, 2013.

[16] A. Venter, E. Joubert, and D. de Beer, "Nutraceutical value of yellow- and red-fleshed South African plums (Prunus salicina Lindl.): evaluation of total antioxidant capacity and phenolic composition," Molecules, vol. 19, no. 3, pp. 30843109, 2014.

[17] M. F. Basanta, A. Marin, S. A. De Leo et al., "Antioxidant Japanese plum (Prunus salicina) microparticles with potential for food preservation," Journal of Functional Foods, vol. 24, pp. 287-296, 2016.

[18] D. Fraternale, "Prunus spinosa fresh fruit juice: antioxidant activity in cell-free and cellular systems," Natural Product Communications, vol. 4, no. 12, pp. 1665-1670, 2009.

[19] B. María Ruiz-Rodríguez, B. de Ancos, C. Sánchez-Moreno et al., "Wild blackthorn (Prunus spinosa L.) and hawthorn (Crataegus monogyna Jacq.) fruits as valuable sources of antioxidants," Fruits, vol. 69, no. 1, pp. 61-73, 2014.

[20] J. Velickovic, D. Kostic, G. Stojanovic et al., "Phenolic composition, antioxidant and antimicrobial activity of the extracts from Prunus spinosa L. fruit," Chemical Industry, vol. 68, no. 3, pp. 297-303, 2014.

[21] S. Meschini, E. Pellegrini, M. Condello et al., "Cytotoxic and apoptotic activities of Prunus spinosa trigno ecotype extract on human cancer cells," Molecules, vol. 22, no. 9, p. 1578, 2017.

[22] R. Slimestad, E. Vangdal, and C. Brede, "Analysis of phenolic compounds in six Norwegian plum cultivars (Prunus domestica L.)," Journal of Agricultural and Food Chemistry, vol. 57, no. 23, pp. 11370-11375, 2009.

[23] V. Usenik, F. Stampar, and R. Veberic, "Anthocyanins and fruit colour in plums (Prunus domestica L.) during ripening," Food Chemistry, vol. 114, no. 2, pp. 529-534, 2009.

[24] D. Treutter, D. Wang, M. A. Farag, G. D. A. Baires, S. Rühmann, and M. Neumüller, "Diversity of phenolic profiles in the fruit skin of prunus domestica plums and related species," Journal of Agricultural and Food Chemistry, vol. 60, no. 48, pp. 12011-12019, 2012.

[25] V. Usenik, F. Stampar, and D. Kastelec, "Phytochemicals in fruits of two Prunus domestica L. plum cultivars during ripening," Journal of the Science of Food and Agriculture, vol. 93, no. 3, pp. 681-692, 2013.

[26] F. V. Dulf, D. C. Vodnar, and C. Socaciu, "Effects of solidstate fermentation with two filamentous fungi on the total phenolic contents, flavonoids, antioxidant activities and lipid fractions of plum fruit (Prunus domestica L.) by-products," Food Chemistry, vol. 209, pp. 27-36, 2016.

[27] M. Turturica, "Effect of thermal treatment on phenolic compounds from plum (Prunus domestica) extracts-a kinetic study," Journal of Food Engineering, vol. 171, pp. 200-207, 2016.

[28] E. Stierlin, S. Azoulay, L. Massi, X. Fernandez, and T. Michel, "Cosmetic potentials of Prunus domestica L. leaves," Journal of the Science of Food and Agriculture, vol. 98, no. 2, pp. 726-736, 2018.

[29] W. Song, S.-T. Qin, F.-X. Fang et al., "Isolation and purification of condensed tannin from the leaves and branches of Prunus cerasifera and its structure and bioactivities," Applied Biochemistry and Biotechnology, vol. 185, no. 2, pp. 464-475, 2018.

[30] R. Pinacho, R. Y. Cavero, I. Astiasarán, D. Ansorena, and M. I. Calvo, "Phenolic compounds of blackthorn (Prunus spinosa L.) and influence of in vitro digestion on their antioxidant capacity," Journal of Functional Foods, vol. 19, pp. 49-62, 2015.

[31] A. C. Irizar, M. F. Fernandez, A. G. González, and A. G. Ravelo, "Constituents of Prunus spinosa," Journal of Natural Products, vol. 55, no. 4, pp. 450-454, 1992.

[32] A. Marchelak, "Bioactivity potential of Prunus spinosa L. Flower extracts: phytochemical profiling, cellular safety, proinflammatory enzymes inhibition and protective effects against oxidative stress in vitro," Frontiers in Pharmacology, vol. 8, p. 680, 2017.

[33] A. Owczarek, A. Magiera, M. Matczak, D. G. Piotrowska, M. A. Olszewska, and A. Marchelak, "Optimisation of preparative HPLC separation of four isomeric kaempferol diglycosides from Prunus spinosa L. by application of the response surface methodology," Phytochemistry Letters, vol. 20, pp. 415-424, 2017.

[34] G. Ozkan, "Phenolic compounds, organic acids, vitamin c and antioxidant capacity in Prunus spinosa," Comptes Rendus De L Academie Bulgare Des Sciences, vol. 72, no. 2, pp. 267-273, 2019.

[35] C. Nunes, S. Guyot, N. Marnet et al., "Characterization of plum procyanidins by thiolytic depolymerization," Journal of Agricultural and Food Chemistry, vol. 56, no. 13, pp. 51885196, 2008.

[36] R. Jaiswal, H. Karaköse, S. Rühmann et al., "Identification of phenolic compounds in plum fruits (Prunus salicina L. And Prunus domestica L.) by high-performance Liquid chromatography/tandem mass spectrometry and characterization of varieties by quantitative phenolic fingerprints," Journal of Agricultural and Food Chemistry, vol. 61, no. 49, pp. 12020-12031, 2013.

[37] F. Celik, M. Gundogdu, S. Alp et al., "Determination of phenolic compounds, antioxidant capacity and organic acids contents of Prunus domestica L., Prunus cerasifera Ehrh. and Prunus spinosa L. fruits by HPLC," Acta Chromatographica, vol. 29, no. 4, pp. 507-510, 2017.

[38] A. Mocan, A. Diuzheva, S. Carradori et al., "Development of novel techniques to extract phenolic compounds from Romanian cultivars of Prunus domestica L. and their biological properties," Food and Chemical Toxicology, vol. 119, pp. 189-198, 2018.

[39] M. Navarro, "Polyphenolic characterization and antioxidant activity of Malus domestica and Prunus domestica cultivars from Costa Rica," Foods, vol. 7, no. 15, pp. 1-19, 2018.

[40] W.-S. Li, H.-B. Hu, Z.-H. Huang, R.-J. Yan, L.-W. Tian, and J. Wu, "Phomopsols A and B from the mangrove endophytic fungus phomopsis sp. xy21: structures, neuroprotective effects, and biogenetic relationships," Organic Letters, vol. 21, no. 19, pp. 7919-7922, 2019.

[41] N. Baltas, "Biochemical properties of partially purified polyphenol oxidase and phenolic compounds of Prunus spinosa L. subsp dasyphylla as measured by HPLC-UV," International Journal of Food Properties, vol. 20, pp. 13771391, 2017.

[42] S.-i. Kayano, N. F. Yamada, T. Suzuki et al., "Quantitative evaluation of antioxidant components in prunes (Prunus domestica L.)," Journal of Agricultural and Food Chemistry, vol. 51, no. 5, pp. 1480-1485, 2003.

[43] H. Kolodziej, M. K. Sakar, J. F. W. Burger, R. Engelshowe, and D. Ferreira, "A-type proanthocyanidins from Prunus spinosa," Phytochemistry, vol. 30, no. 6, pp. 2041-2047, 1991.

[44] A. G. Gonzalez, "Type a proanthocyanidins from Prunus spinosa," Phytochemistry, vol. 31, no. 4, pp. 1432-1434, 1992. 
[45] M. Alejandre, D. Ansorena, M. I. Calvo, R. Y. Cavero, and I. Astiasarán, "Influence of a gel emulsion containing microalgal oil and a blackthorn (Prunus spinosa L.) branch extract on the antioxidant capacity and acceptability of reduced-fat beef patties," Meat Science, vol. 148, pp. 219-222, 2019.

[46] Y. Kimura, H. Ito, M. Kawaji, T. Ikami, and T. Hatano, "Characterization and antioxidative properties of oligomeric proanthocyanidin from prunes, dried fruit of Prunus domestica L." Bioscience, Biotechnology, and Biochemistry, vol. 72, no. 6, pp. 1615-1618, 2008.

[47] M. Olszewska and M. Wolbis, "Flavonoids from the leaves of Prunus spinosa L." Polish Journal of Chemistry, vol. 76, no. 7, pp. 967-974, 2002.

[48] G. Lombardi-Boccia, M. Lucarini, S. Lanzi, A. Aguzzi, and M. Cappelloni, "Nutrients and antioxidant molecules in yellow plums (Prunus domestica L.) from conventional and organic productions: a comparative study," Journal of Agricultural and Food Chemistry, vol. 52, no. 1, pp. 90-94, 2004.

[49] H. S. El-Beltagi, "Evaluation of the phytochemical, antioxidant, antibacterial and anticancer activity of prunus domestica fruit," Notulae Botanicae Horti Agrobotanici ClujNapoca, vol. 47, no. 2, pp. 395-404, 2019.

[50] W. Qiang, "Chemical constituents extracted from leaves of Prunus cerasifera Ehrh. cv.atropurpurea jacg. and their antioxidant activities in vitro," Chemistry and Industry of Forest Products, vol. 35, no. 5, pp. 116-122, 2015.

[51] N. Nakatani, S.-i. Kayano, H. Kikuzaki, K. Sumino, K. Katagiri, and T. Mitani, "Identification, quantitative determination, and antioxidative activities of chlorogenic acid isomers in prune (Prunus domestica L.)," Journal of Agricultural and Food Chemistry, vol. 48, no. 11, pp. 5512-5516, 2000.

[52] S. I. Kayano, "Antioxidant properties of prunes (Prunus domestica L.) and their constituents," Biofactors, vol. 21, no. 1-4, pp. 309-313, 2004.

[53] F. Khallouki, R. Haubner, G. Erben, C. M. Ulrich, and R. W. Owen, "Phytochemical composition and antioxidant capacity of various botanical parts of the fruits of Prunus $\times$ domestica L. from the Lorraine region of Europe," Food Chemistry, vol. 133, no. 3, pp. 697-706, 2012.

[54] N. Miletic, "Polyphenolic content and antioxidant capacity in fruits of plum (Prunus domestica L.) cultivars "valjevka" and "mildora" as influenced by air drying," Journal of Food Quality, vol. 36, no. 4, pp. 229-237, 2013.

[55] M. Bose, "Identification of a flavonoid isolated from plum (Prunus domestica) as a potent inhibitor of Hepatitis C virus entry," Scientific Reports, vol. 7, p. 3965, 2017.

[56] L. Wei, "Study on the flavonoids in the leaves of prunus cerasifera grown in Xinjiang," Modern Food Science and Technology, vol. 34, no. 9, pp. 269-273, 2018.

[57] G. R. Nagarajan and T. R. Seshadri, "Flavonoid components of the heartwood of Prunus domestica linn," Phytochemistry, vol. 3, no. 4, pp. 477-484, 1964.

[58] A. Mahmood, I. Fatima, S. Kosar, R. Ahmed, and A. Malik, "Structural determination of prunusins A and B, new C-alkylated flavonoids from Prunus domestica, by 1D and 2D NMR spectroscopy," Magnetic Resonance in Chemistry: $M R C$, vol. 48, no. 2, pp. 151-154, 2010.

[59] S. Kosar, "Prudomestisides A and B, new flavonoidal glucosides from Prunus domestica and their antioxidant activity," Journal of the Chemical Society of Pakistan, vol. 35, no. 1, pp. 169-174, 2013.

[60] V. S. Parmar, "Dihydroflavonols from Prunus domestica," Phytochemistry, vol. 31, no. 6, pp. 2185-2186, 1992.
[61] S. Kosar, I. Fatima, A. Mahmood et al., "Purunusides A-C, $\alpha$-glucosidase inhibitory homoisoflavone glucosides from Prunus domestica," Archives of Pharmacal Research, vol. 32, no. 12, pp. 1705-1710, 2009.

[62] R. Aliyazicioglu, O. Yildiz, H. Sahin et al., "Phenolic components and antioxidant activity of Prunus spinosa from gumushane, Turkey," Chemistry of Natural Compounds, vol. 51, no. 2, pp. 346-349, 2015.

[63] S.-i. Kayano, H. Kikuzaki, T. Ikami, T. Suzuki, T. Mitani, and N. Nakatani, "A new bipyrrole and some phenolic constituents in prunes (Prunus domestica L.) and their oxygen radical absorbance capacity (ORAC)," Bioscience, Biotechnology, and Biochemistry, vol. 68, no. 4, pp. 942-944, 2004.

[64] J. Bouayed, "Chlorogenic acid, a polyphenol from Prunus domestica (Mirabelle), with coupled anxiolytic and antioxidant effects," Journal of the Neurological Sciences, vol. 262, no. 1-2, pp. 77-84, 2007.

[65] A. Marchelak, A. Owczarek, M. Rutkowska et al., "New insights into antioxidant activity of Prunus spinosa flowers: extracts, model polyphenols and their phenolic metabolites in plasma towards multiple in vivo-relevant oxidants," Phytochemistry Letters, vol. 30, pp. 288-295, 2019.

[66] H. Kikuzaki, S.-i. Kayano, N. Fukutsuka et al., "Abscisic acid related compounds and lignans in prunes (Prunus domestica L.) and their oxygen radical absorbance capacity (ORAC)," Journal of Agricultural and Food Chemistry, vol. 52, no. 2, pp. 344-349, 2004.

[67] Y. Kumarasamy, P. J. Cox, M. Jaspars, L. Nahar, and S. D. Sarker, "Cyanogenic glycosids from Prunus spinosa (Rosaceae)," Biochemical Systematics and Ecology, vol. 31, no. 9, pp. 1063-1065, 2003.

[68] G. R. Nagarajan and V. S. Parmar, "Phloracetophenone derivatives in Prunus domestica," Phytochemistry, vol. 16, no. 5, pp. 614-615, 1977.

[69] S.-i. Kayano, H. Kikuzaki, S. Hashimoto, K. Kasamatsu, T. Ikami, and N. Nakatani, "Glucosyl terpenates from the dried fruits of Prunus domestica L," Phytochemistry Letters, vol. 8, pp. 132-136, 2014.

[70] X. Qiao-Lin, X. Hai-hui, and Y. Masayuki, "Chemical constituents from the seeds of prunus domestica L," Journal of Tropical and Subtropical Botany, vol. 20, no. 3, pp. 299302, 2012.

[71] P. Birwal, "Plums: a brief introduction," Journal of Food, Nutrition and Population Health, vol. 1, no. 1, pp. 1-5, 2017.

[72] S. B. Jaffri and K. S. Ahmad, "Prunus cerasifera Ehrh. fabricated $\mathrm{ZnO}$ nano falcates and its photocatalytic and dose dependent in vitro bio-activity," Open Chemistry, vol. 16, no. 1, pp. 141-154, 2018.

[73] K. Gündüz and O. Saraçoğlu, "Variation in total phenolic content and antioxidant activity of Prunus cerasifera Ehrh. selections from Mediterranean region of Turkey," Scientia Horticulturae, vol. 134, pp. 88-92, 2012.

[74] N. Miletic, "Phenolic content and antioxidant capacity of fruits of plum cv.'Stanley'('Prunus domestica'L.) as influenced by maturity stage and on-tree ripening," Australian Journal of Crop Science, vol. 6, no. 4, pp. 681-687, 2012.

[75] Y. Hu, J. Meng, and B. Hu, "Research of antioxidant activity and stability of the extract from Pranus cerasifera Ehrh. Cv. Atropurpurea leaves," Food Science, vol. 23, pp. 274-276, 2002.

[76] B. Zhang, Q. Liu, and H. Zhanf, "Optimization of the ultrasonic extraction of anthocyanins from Prunus cerasifera leares," Journal of Shanghai Jiaotong University-Agricultural Science, vol. 31, no. 6, pp. 41-47, 2013. 
[77] M. Hou, Y. Sun, and H. Yu, "Extraction and stability of red pigments from Pranus cerasifera Ehrh. leaves," Science \& Technology Information, vol. 13, p. 31, 2011.

[78] Y. Gao, "Chromogenic pigments in Prunus cerasifera leaves," Journal of Zhejiang A\&F University, vol. 31, no. 3, pp. 481487, 2014.

[79] M. Norton, Growing Prunes (Dried Plums) in California: An Overview, UCANR Publications, Davis, CA, USA, 2007.

[80] R. N. Chopra, S. L. Nayar, and I. C. Chopra, Glossary of Indian Medicinal Plants, vol. 1, Council of Scientific \& Industrial Research, New Delhi, India, 1956.

[81] M. Stacewicz-Sapuntzakis, P. E. Bowen, E. A. Hussain, B. I. Damayanti-Wood, and N. R. Farnsworth, "Chemical composition and potential health effects of Prunes: a functional food?" Critical Reviews in Food Science and Nutrition, vol. 41, no. 4, pp. 251-286, 2001.

[82] L. F. Tinker, B. O. Schneeman, P. A. Davis, D. D. Gallaher, and C. R. Waggoner, "Consumption of prunes as a source of dietary fiber in men with mild hypercholesterolemia," The American Journal of Clinical Nutrition, vol. 53, no. 5, pp. 1259-1265, 1991.

[83] M. H. Yu, H. G. Im, H.-I. Kim, and I.-S. Lee, "Induction of apoptosis by immature plum in human hepatocellular carcinoma," Journal of Medicinal Food, vol. 12, no. 3, pp. 518-527, 2009.

[84] G. Noratto, W. Porter, D. Byrne, and L. Cisneros-Zevallos, "Identifying peach and plum polyphenols with chemopreventive potential against estrogen-independent breast cancer cells," Journal of Agricultural and Food Chemistry, vol. 57, no. 12, pp. 5219-5226, 2009.

[85] M. Franklin, S. Y. Bu, M. R. Lerner et al., "Dried plum prevents bone loss in a male osteoporosis model via IGF-I and the RANK pathway," Bone, vol. 39, no. 6, pp. 1331-1342, 2006.

[86] J. W. Pawlowski, B. R. Martin, G. P. McCabe, M. G. Ferruzzi, and C. M. Weaver, "Plum and soy aglycon extracts superior at increasing bone calcium retention in ovariectomized Sprague Dawley rats," Journal of Agricultural and Food Chemistry, vol. 62, no. 26, pp. 6108-6117, 2014.

[87] B. Shukitt-Hale, W. Kalt, A. N. Carey, M. Vinqvist-Tymchuk, J. McDonald, and J. A. Joseph, "Plum juice, but not dried plum powder, is effective in mitigating cognitive deficits in aged rats," Nutrition, vol. 25, no. 5, pp. 567-573, 2009.

[88] S. Shahidi, S. Setareye, and M. Mahmoodi, "Effect of Prunus domestica L. (mirabelle) on learning and memory in mice," Ancient Science of Life, vol. 32, no. 3, pp. 139-143, 2013.

[89] E. Rendina, Y. F. Lim, D. Marlow et al., "Dietary supplementation with dried plum prevents ovariectomy-induced bone loss while modulating the immune response in C57BL/ 6J mice," The Journal of Nutritional Biochemistry, vol. 23, no. 1, pp. 60-68, 2012.

[90] L. K. Kim DO, O. K. Chun, H. J. Leer, and C. Y. Lee, "Antiproliferative activity of polyphenolics in plums," Food Science and Biotechnology, vol. 12, pp. 399-402, 2003.

[91] T. Fujii, T. Ikami, J.-W. Xu, and K. Ikeda, "Prune extract (Prunus domestica L.) suppresses the proliferation and induces the apoptosis of human colon carcinoma Caco-2," Journal of Nutritional Science and Vitaminology, vol. 52, no. 5, pp. 389-391, 2006.

[92] M. A. Lea, "Inhibition of growth and induction of differentiation of colon cancer cells by peach and plum phenolic compounds," Anticancer Research, vol. 28, no. 4B, pp. 2067-2076, 2008.

[93] S. Hooshmand, A. Kumar, J. Y. Zhang, S. A. Johnson, S. C. Chai, and B. H. Arjmandi, "Evidence for anti- inflammatory and antioxidative properties of dried plum polyphenols in macrophage RAW 264.7 cells," Food \& Function, vol. 6, no. 5, pp. 1719-1725, 2015.

[94] T. M. Cantu-Jungles, D. Maria-Ferreira, L. M. da Silva et al., "Polysaccharides from prunes: gastroprotective activity and structural elucidation of bioactive pectins," Food Chemistry, vol. 146, pp. 492-499, 2014.

[95] N. Mishra, N. S. Gill, A. Mishra, S. Mishra, A. Shukla, and A. Upadhayay, "Evaluation of antioxidant and antiulcer potentials of Prunus domestica fruit methanolic and extract on wistar albino rats," Journal of Pharmacology and Toxicology, vol. 7, no. 6, pp. 305-311, 2012.

[96] J. Bouayed, H. Rammal, A. Dicko, C. Younos, and R. Soulimani, "The antioxidant effect of plums and polyphenolic compounds against $\mathrm{H}_{2} \mathrm{O}_{2}$-induced oxidative stress in mouse blood granulocytes," Journal of Medicinal Food, vol. 12, no. 4, pp. 861-868, 2009.

[97] T. Ahmed, "Use of prunes as a control of hypertension," Journal of Ayub Medical College Abbottabad, vol. 22, no. 1, pp. 28-31, 2010.

[98] L. Piirainen, K. Peuhkuri, K. Bäckström, R. Korpela, and S. Salminen, "Prune juice has a mild laxative effect in adults with certain gastrointestinal symptoms," Nutrition Research, vol. 27, no. 8, pp. 511-513, 2007.

[99] K. Karasawa, R. Miyashita, and H. Otani, "Anti-allergic properties of a fruit extract of prune (Prunus domestica L.) in mite-sensitized BALB/c mice," Food Science and Technology Research, vol. 18, no. 5, pp. 755-760, 2012.

[100] P.-H. Kuo, C.-I. Lin, Y.-H. Chen, W.-C. Chiu, and S.-H. Lin, "A high-cholesterol diet enriched with polyphenols from Oriental plums (Prunus salicina) improves cognitive function and lowers brain cholesterol levels and neurodegenerative-related protein expression in mice," British Journal of Nutrition, vol. 113, no. 10, pp. 1550-1557, 2015.

[101] M. Hee Yu, H. Gwon Im, S.-O. Lee, C. Sung, D.-C. Park, and I.-S. Lee, "Induction of apoptosis by immature fruits of Prunus salicina Lindl. cv. Soldam in MDA-MB-231 human breast cancer cells," International Journal of Food Sciences and Nutrition, vol. 58, no. 1, pp. 42-53, 2007.

[102] G. Noratto, H. S. D. Martino, S. Simbo, D. Byrne, and S. U. Mertens-Talcott, "Consumption of polyphenol-rich peach and plum juice prevents risk factors for obesity-related metabolic disorders and cardiovascular disease in Zucker rats," The Journal of Nutritional Biochemistry, vol. 26, no. 6, pp. 633-641, 2015.

[103] T. Tutin, 1980: Flora Europaea, University Press, Cambridge, UK, 1964

[104] A. Mohanty, J. P. Martín, and I. Aguinagalde, "Population genetic analysis of European Prunus spinosa (Rosaceae) using chloroplast DNA markers," American Journal of Botany, vol. 89, no. 8, pp. 1223-1228, 2002.

[105] M. Sakar and H. Kolodziej, "Flavonoid glycosides from the flowers of Prunus spinosa," Fitoterapia, vol. 64, no. 2, pp. 180-181, 1993.

[106] M. Olszewska and M. Wolbis, "Flavonoids from the flowers of Prunus spinosa L," Acta Poloniae Pharmaceutica, vol. 58, no. 5, pp. 367-372, 2001.

[107] M. Olszewska and M. Wolbiśs, "Further flavonoids from the flowers of Prunus spinosa L," Acta Poloniae Pharmaceutica, vol. 59, no. 2, pp. 133-137, 2002.

[108] R. Guimarães, L. Barros, M. Dueñas et al., "Characterisation of phenolic compounds in wild fruits from Northeastern Portugal," Food Chemistry, vol. 141, no. 4, pp. 3721-3730, 2013. 